\title{
DYNAMIC ANALYSIS OF A REWARD PROCESS DEFINED ON A CYCLIC RENEWAL PROCESS WITH APPLICATIONS TO PREVENTIVE MAINTENANCE PROBLEMS
}

\author{
Ushio Sumita Kazuki Takahashi \\ University of Tsukuba
}

(Received June 11, 2007; Revised October 27, 2008)

\begin{abstract}
A cyclic renewal process is considered as an extension of an alternating renewal process, where each of the underlying independently and identically distributed (i.i.d.) nonnegative random increments is composed of multiple stages. Such a process may be appropriate for analyzing optimal preventive maintenance policies for production management, where a pair of two stages representing an uptime until a minor failure and the subsequent minimal repair time would be repeated until it is decided to conduct a complete overhaul. In order to address economic problems in such applications, we also introduce a reward process with jumps defined on the cyclic renewal process. When the system is running in stage $j$, the profit grows linearly at the rate of $\rho(j)$. Upon a minor failure, the subsequent minimal repair in stage $(j+1)$ incurs the linear cost at the rate of $\rho(j+1)$. In addition, the fixed cost may be imposed whenever either a minimal repair or a complete overhaul takes place, resulting in jumps of the reward process. The problem is then to determine when to conduct a complete overhaul so as to maximize the total reward in the time interval $(0, T]$. A multivariate Markov process generated from both the cyclic renewal process and the reward process is studied extensively, yielding various new transform results explicitly and deriving their asymptotic expansions. These results are used to numerically explore optimal preventive maintenance policies for production management.
\end{abstract}

Keywords: Applied probability, cyclic renewal process, preventive maintenance

\section{Introduction}

Renewal theory is the branch of probability theory concerning a variety of problems related to the partial sums of a sequence of i.i.d. nonnegative random variables. More specifically, let $\left(Y_{n}\right)_{n=1}^{\infty}$ be a sequence of i.i.d. nonnegative random variables and define $S_{n}=\sum_{j=1}^{n} Y_{j}$. The renewal process $\{N(t) ; t \geqslant 0\}$ associated with $\left(Y_{n}\right)_{n=1}^{\infty}$ is a counting process defined by $N(t)=n$ if and only if $S_{n} \leqslant t<S_{n+1}$. Of interest are the renewal function $H(t)=\mathrm{E}[N(t)]$, the renewal density $h(t)=\frac{d}{d t} H(t)$ if it exists, and other related probabilistic entities. As the name "renewal theory" indicates, the study stemmed from a class of applications involving successive replacements of items subject to failure. Here, $Y_{n}$ denotes the lifetime of the $n$-th item and $N(t)$ is the number of replacements that took place by time $t$.

The renewal theory has been extended in many ways. A delayed renewal process, for example, has the distribution of $Y_{1}$ different from that of $Y_{n}(n>1)$, and an alternating renewal process deals with a situation where $Y_{n}$ consists of two stages : the system uptime and the system repair time, see e.g. Cox [4]. A Markov renewal process considers a case where distributions of interfailure times are governed by a Markov chain $\{J(n) ; n=0,1,2, \cdots\}$ in discrete time, i.e. if $J(n-1)=i$ and $J(n)=j$, then the distribution of $Y_{n}$ is given by $A_{i j}(x)$. The reader is referred to Keilson [12], Keilson and Rao [13, 14], and an excellent 
survey paper by Cinlar [2] for the study of Markov renewal processes. Keener [11] develops a general renewal theory where i.i.d. increments have support on full continuum. In Kijima and Sumita [15], the renewal theory is extended in that the distribution of $Y_{n+1}$ depends on the partial sum $S_{n}$ up to the $n$-th increment.

The purpose of this paper is to introduce a cyclic renewal process as an extension of an alternating renewal process, where each of the underlying i.i.d. nonnegative random increments is composed of $J$ stages, i.e. $Y_{n}=\sum_{j=1}^{J} X_{n: j}, n \geqslant 1$, where $X_{n: j}$ are i.i.d. with respect to $n$ and consequently so are $Y_{n}$. Such a process may be appropriate for analyzing optimal preventive maintenance policies for production management, where a pair of two stages representing an uptime until a minor failure and the subsequent minimal repair time would be repeated until it is decided to conduct a complete overhaul. In order to address economic problems in such applications, we also introduce a reward process with jumps defined on the cyclic renewal process. When the system is running in stage $j$, the profit grows linearly at the rate of $\rho(j)$. Upon a minor failure, the subsequent minimal repair in stage $(j+1)$ incurs the linear cost at the rate of $\rho(j+1)$. In addition, the fixed cost may be imposed whenever either a minimal repair or a complete overhaul takes place, resulting in jumps of the reward process. The problem is then to determine when to conduct a complete overhaul so as to maximize the total reward in the time interval $(0, T]$.

When the renewal aspect is suppressed, the above model is reduced to a semi-Markov process. The study of semi-Markov processes dates back to the middle of $1950 \mathrm{~s}$, originated by works of Lévy [16], Smith [25] and Takács [29]. Subsequently the scope of the study has been expanded through a series of papers by Pyke [22,23], Pyke and Schaufele [24], and Moore and Pyke [21]. Since the early 1960s, the field attracted many researchers resulting in a collection of quite extensive results. The reader is referred to two excellent survey papers by Cinlar $[1,2]$ and references therein for extensive analysis of semi-Markov and related processes. Reward processes defined on semi-Markov processes also have been studied extensively, including the original works by Jewell [8-10] followed by Mclean and Neuts [20], Hunter [6], Howard [5], Çinlar [3], Sumita and Masuda [27], Sumita, Kubat and Masuda [26], Sumita, Masuda and Kubat [28], Masuda and Sumita [19] and Igaki, Sumita and Kowada [7] to name a few. However, to the best knowledge of the authors, the joint distribution of the cyclic renewal process, the underlying semi-Markov process and the reward process has never been studied in the literature.

In this paper, a multivariate Markov process generated from both the cyclic renewal process and the reward process is studied extensively, yielding various new transform results explicitly and deriving their asymptotic expansions. These results are used to numerically explore optimal preventive maintenance policies for production management. It is worth noting that, while the long-term analysis can be done by combining the individual cyclic components into a single lifetime and working out the resulting classical renewal process, this simplified approach can offer only the approximated dynamic optimal strategy in $(0, T]$. The exact dynamic optimal strategy in $(0, T]$ can be derived only through the detailed analysis of the underlying cyclic renewal process. The danger of exclusive reliance on the longterm analysis or the classical renewal approximation will be demonstrated by exhibiting numerically the differences between the long-term optimal strategy or the approximated dynamic optimal strategy and the exact dynamic optimal strategy.

The structure of this paper is as follows. A cyclic renewal process $\{N(t) ; t \geqslant 0\}$ is formally introduced in Section 2 based on a cyclic semi-Markov process $\{J(t) ; t \geqslant 0\}$ describing multiple stages to constitute system lifetimes. The associated age process $\{X(t) ; t \geqslant 0\}$ and the reward process $\{Z(t) ; t \geqslant 0\}$ are also introduced so that the multivariate process 
$[N(t), J(t), X(t), Z(t)]$ becomes Markov. Section 3 is devoted to analysis of this multivariate process by examining its probabilistic flow in its state space, yielding various new transform results. In Section 4, the asymptotic expansions of the expectation $\mathrm{E}[Z(t)]$ and the correlation Cor $[N(t), Z(t)]$ as $t \rightarrow \infty$ are derived. We discuss the differences between the long-term optimal strategy or the approximated dynamic optimal strategy and the exact dynamic optimal strategy in Section 5. In Section 6, the results obtained in Section 4 are used to numerically explore optimal preventive maintenance policies for production management. Some mathematical details are deferred to Appendix for enhancing the readability of the paper.

\section{Model Description}

We consider a cyclic renewal process $\{N(t) ; t \geqslant 0\}$ defined on $\mathcal{N}=\{0,1,2, \cdots\}$ where the underlying lifetime consists of $J$ stages and $N(t)$ denotes the number of failures by time $t$. More specifically, let $\mathcal{J}=\{1,2, \cdots, J\}$ be the set of the stages and let the dwell time in stage $j \in \mathcal{J}$ be a nonnegative random variable denoted by $X_{j}$. Throughout the paper, we assume that $X_{j}(j \in \mathcal{J})$ are independent of the failure count and also mutually independent. For each $j \in \mathcal{J}$, it is assumed that $X_{j}$ is absolutely continuous characterized by

$$
\bar{A}_{j}(x) \stackrel{\text { def }}{=} \mathrm{P}\left[X_{j}>x\right] ; a_{j}(x) \stackrel{\text { def }}{=}-\frac{d}{d x} \bar{A}_{j}(x) ; \eta_{j}(x) \stackrel{\text { def }}{=} \frac{a_{j}(x)}{\bar{A}_{j}(x)} ; \alpha_{j}(v) \stackrel{\text { def }}{=} \int_{0}^{\infty} e^{-v x} a_{j}(x) d x,
$$

where $\bar{A}_{j}(x), a_{j}(x), \eta_{j}(x)$ and $\alpha_{j}(v)$ are the survival function, the probability density function, the hazard function and the Laplace transform of $a_{j}(x)$ respectively. Here $v$ takes values from the complex plane satisfying $R e(v)>0$ so that $\alpha_{j}(v)$ is well defined. A lifetime associated with the cyclic renewal process is given by

$$
Y=\sum_{j=1}^{J} X_{j}
$$

Let $Y_{k}$ be the lifetime of the $k$-th renewal cycle where $Y_{k}$ 's are i.i.d. with common structure of $(2.2)$. For $k=0$, one then sees that $\mathrm{P}[N(t)=0]=\mathrm{P}\left[0 \leqslant t<Y_{1}\right]$ and for $k \geqslant 1$, $\mathrm{P}[N(t)=k]=\mathrm{P}\left[\sum_{m=1}^{k} Y_{m} \leqslant t<\sum_{m=1}^{k+1} Y_{m}\right]$.

Let $\{J(t) ; t \geqslant 0\}$ be a stochastic process describing the stage at time $t$. We note that $J(t)$ is a cyclic semi-Markov process on $\mathcal{J}=\{1, \cdots, J\}$ governed by the matrix distribution function $\underline{\underline{A}}(x)$ where

$$
\underline{A}(x) \stackrel{\text { def }}{=}\left[\begin{array}{ccccc}
0 & A_{1}(x) & 0 & \cdots & 0 \\
0 & 0 & A_{2}(x) & \cdots & 0 \\
0 & 0 & \cdots & \ddots & \vdots \\
0 & 0 & \cdots & 0 & A_{J-1}(x) \\
A_{J}(x) & 0 & \cdots & 0 & 0
\end{array}\right] ; A_{j}(x) \stackrel{\text { def }}{=} 1-\bar{A}_{j}(x) .
$$

Since the bivariate process $[N(t), J(t)]$ is not Markov, we introduce an additional process $\{X(t) ; t \geqslant 0\}$ on $\mathcal{R}^{+}$denoting the elapsed time since the last entry into the current stage at time $t$, where $\mathcal{R}^{+}$is the set of nonnegative real numbers. This process is called the age process. The trivariate process $[N(t), J(t), X(t)]$ then becomes Markov. A typical sample path of $[N(t), J(t), X(t)]$ is depicted in Figure 2.1 where $N(0)=0, J(0)=i$ and $X(0)=0$.

From an application point of view, of particular interest is a reward process $\{Z(t) ; t \geqslant 0\}$ with jumps defined on $[N(t), J(t), X(t)]$. We assume that the reward increases or decreases 

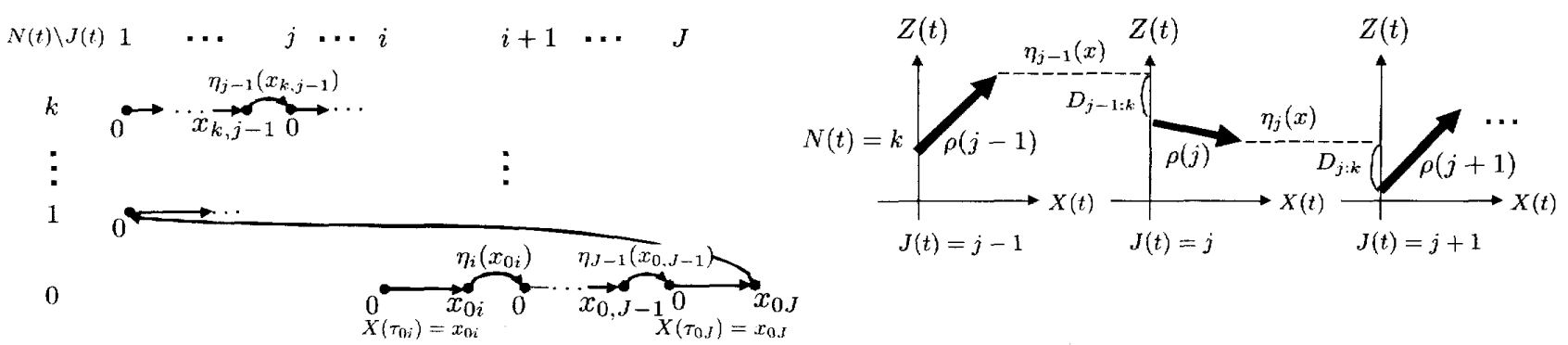

Figure 2.1: Typical sample path of $[N(t)$, $J(t), X(t)]$

Figure 2.2: Typical sample path of $[X(t), Z(t), J(t)]$ with jumps

linearly at the rate of $\rho(j)$ when $J(t)$ is in state $j \in \mathcal{J}$. Furthermore, the reward process jumps in the random amount of $D_{j}$ when $J(t)$ moves from $j$ to $j+1$ for $j \in \mathcal{J} \backslash\{J\}$, and $D_{J}$ for a transition from $J$ to 1 . Accordingly, $Z(t)$ takes a value from $\mathcal{R}$ where $\mathcal{R}$ is the set of real numbers. As for $X_{j}(j \in \mathcal{J})$, it is assumed that $D_{j}(j \in \mathcal{J})$ are independent of the failure count, mutually independent, and absolutely continuous having

$$
\bar{B}_{j}(z) \stackrel{\text { def }}{=} \mathrm{P}\left[D_{j}>z\right] ; b_{j}(z) \stackrel{\text { def }}{=}-\frac{d}{d z} \bar{B}_{j}(z) ; \beta_{j}(w) \stackrel{\text { def }}{=} \int_{-\infty}^{\infty} e^{-w z} b_{j}(z) d z
$$

where $w$ takes values on the unit circle on the complex plane so that $\beta_{j}(w)$ is well defined.

In order to describe the reward process $\{Z(t) ; t \geqslant 0\}$ more formally, let $\left\{M_{j}(t) ; t \geqslant 0\right\}$ be the stochastic process counting the number of transitions of $J(t)$ from $j$ to $j+1$ by time $t$ for $j \in \mathcal{J} \backslash\{J\}$. The stochastic process $\left\{M_{J}(t) ; t \geqslant 0\right\}$ is defined similarly for transitions of $J(t)$ from $J$ to 1 . One then has

$$
Z(t)=\int_{0}^{t} \rho(J(\tau)) d \tau+\sum_{j=1}^{J} \sum_{m=1}^{M_{j}(t)} D_{j: m},
$$

where $D_{j: m}$ denotes the jump amount associated with the $m$-th transition from $j$ to $j+1$ for $j \in \mathcal{J} \backslash\{J\}$, and from $J$ to 1 for $j=J$. Following the mathematical convention, we define $\sum_{m=a}^{b} c_{m}=0$ whenever $a>b$. It should be noted that, by the assumptions discussed above, $D_{j: m}\left(m=1, \cdots, M_{j}(t)\right)$ are i.i.d. with respect to $m$. When $J(t)$ is a general semiMarkov process, the expectation of the semi-Markov reward process with jumps is given in Howard [5]. The transform results of $[J(t), Z(t)]$ are derived in McLean and Neuts [20]. The trivariate Markov process $[J(t), X(t), Z(t)]$ is also studied in detail in Sumita and Masuda $[26,27]$ and Masuda [18]. The thrust of this paper is to analyze the multivariate process $[N(t), J(t), X(t), Z(t)]$ where the cyclic renewal process $N(t)$ is incorporated together with $[J(t), X(t), Z(t)]$, which is new. The results are then used to numerically explore optimal preventive maintenance policies for production management.

\section{Dynamic Analysis of Multivariate Process $[N(t), J(t), X(t), Z(t)]$}

In this section, we analyze the multivariate process $[N(t), J(t), X(t), Z(t)]$ by describing its probabilistic flow in the state space $\mathcal{N} \times \mathcal{J} \times \mathcal{R}^{+} \times \mathcal{R}$. For this purpose, let $F_{k: i j}(x, z, t)$ be the joint distribution function of $[N(t), J(t), X(t), Z(t)]$ given $J(0)=i, X(0)=Z(0)=0$. More formally, we define

$$
\begin{array}{ll} 
& F_{k: i j}(x, z, t) \\
\stackrel{\text { def }}{=} & \mathrm{P}[N(t)=k, J(t)=j, X(t) \leqslant x, Z(t) \leqslant z \mid J(0)=i, X(0)=Z(0)=0] .
\end{array}
$$


The corresponding joint probability density function is given by

$$
f_{k: i j}(x, z, t) \stackrel{\text { def }}{=} \frac{\partial^{2}}{\partial x \partial z} F_{k: i j}(x, z, t)
$$

For the process $[N(t), J(t), X(t), Z(t)]$ to be at $(0, j, x, z)$ at time $t>0$ given $J(0)=i$, either no transition of $J(t)$ has occurred in the time interval $[0, t]$ with $j=i$, or at least one transition of $J(t)$ from $J(0)=i$ occurred in $[0, t)$, the process entered the state $(0, j, 0+, z-$ $\rho(j) x)$ at time $t-x$, and no transition of $J(t)$ has occurred since then. Accordingly, one has

$$
\begin{aligned}
f_{0: i j}(x, z, t) & =\delta_{\{j=i\}} \delta(z-\rho(j) t) \delta(t-x) \bar{A}_{j}(x) \\
& +\delta_{\{j>i\}} f_{0: i j}(0+, z-\rho(j) x, t-x) \bar{A}_{j}(x), x>0, j=i, \cdots, J
\end{aligned}
$$

Here, $\delta_{\{P\}}=1$ if the statement $P$ holds true, $\delta_{\{P\}}=0$ otherwise, and $\delta(t)$ is the delta function defined as the unit function associated with the convolution operation, i.e., $f(x)=$ $\int f(y) \delta(x-y) d y$ for any integrable function $f$. Similarly, for $k>0$, to be at $(k, j, x, z)$ at time $t>0$, the process should have entered the state $(k, j, 0+, z-\rho(j) x)$ at time $t-x$ and no transition of $J(t)$ has occurred since then. This then yields

$$
f_{k: i j}(x, z, t)=f_{k: i j}(0+, z-\rho(j) x, t-x) \bar{A}_{j}(x), \quad x>0, k \geqslant 1 .
$$

In order to determine the boundary conditions $f_{k: i j}(0+, z, t)$ associated with the age process $X(t)$, we first consider the case that $k=0, z=0+$ and $t=0+$. One then sees that $f_{0: i j}(0+, 0+, 0+)=\delta_{\{j=i\}} \delta(z) \delta(t)$. For $t>0$ and $j \geqslant i$, the process $[N(t), J(t), X(t), Z(t)]$ just enters the state $(0, j, 0+, z)$ at time $t$ only if the dwell time of $J(t)$ in state $j-1$ expires at time $t$ with the reward at $z-D_{j-1}$ followed by the instantaneous jump of size $D_{j-1}$ so that $Z(t)=z$. Combining the two cases, one observes that

$$
\begin{aligned}
& f_{0: i j}(0+, z, t)=\delta_{\{j=i\}} \delta(z) \delta(t) \\
+ & \delta_{\{j>i\}} \int_{0}^{\infty} \int_{-\infty}^{\infty} f_{0: i, j-1}\left(x, z-z^{\prime}, t\right) \eta_{j-1}(x) b_{j-1}\left(z^{\prime}\right) d z^{\prime} d x, j=i, \cdots, J .
\end{aligned}
$$

For $k \geqslant 1$, similar a guments lead to

$$
f_{k: i j}(0+, z, t)=\varangle \begin{array}{ll}
\int_{0}^{\infty} \int_{-\infty}^{\infty} f_{k-1: i J}\left(x, z-z^{\prime}, t\right) \eta_{J}(x) b_{J}\left(z^{\prime}\right) d z^{\prime} d x, & j=1 \\
\int_{0}^{\infty} \int_{-\infty}^{\infty} f_{k: i, j-1}\left(x, z-z^{\prime}, t\right) \eta_{j-1}(x) b_{j-1}\left(z^{\prime}\right) d z^{\prime} d x, & 2 \leqslant j \leqslant J
\end{array} .
$$

We are now in a position to prove the key theorem of this paper. For notational convenience, the following matrix Laplace-Fourier transforms are introduced.

$$
\begin{aligned}
& \underline{\underline{\varphi}}_{k}(x, z, s) \stackrel{\text { def }}{=}\left[\hat{\varphi}_{k: i j}(x, z, s)\right] ; \hat{\varphi}_{k: i j}(x, z, s) \stackrel{\text { def }}{=} \int_{0}^{\infty} e^{-s t} f_{k: i j}(x, z, t) d t \\
& \underline{\hat{\varphi}}_{k}(x, w, s) \stackrel{\text { def }}{=}\left[\hat{\hat{\varphi}}_{k: i j}(x, w, s)\right] ; \hat{\hat{\varphi}}_{k: i j}(x, w, s) \stackrel{\text { def }}{=} \int_{-\infty}^{\infty} e^{-w z} \hat{\varphi}_{k: i j}(x, z, s) d z \\
& \underline{\hat{\hat{\varphi}}}_{k}(v, w, s) \stackrel{\text { def }}{=}\left[\hat{\hat{\varphi}}_{k: i j}(v, w, s)\right] ; \hat{\hat{\varphi}}_{k: i j}(v, w, s) \stackrel{\text { def }}{=} \int_{0}^{\infty} e^{-v x} \hat{\hat{\varphi}}_{k: i j}(x, w, s) d x \\
& \underline{\underline{\xi}}_{k}(0+, z, s) \stackrel{\text { def }}{=}\left[\hat{\xi}_{k: i j}(0+, z, s)\right] ; \hat{\xi}_{k: i j}(0+, z, s) \stackrel{\text { def }}{=} \int_{0}^{\infty} e^{-s t} f_{k: i j}(0+, z, t) d t \\
& \underline{\hat{\xi}}_{k}(0+, w, s) \stackrel{\text { def }}{=}\left[\hat{\hat{\xi}}_{k: i j}(0+, w, s)\right] ; \hat{\hat{\xi}}_{k: i j}(0+, w, s) \stackrel{\text { def }}{=} \int_{-\infty}^{\infty} e^{-w z} \hat{\xi}_{k: i j}(0+, z, s) d z,
\end{aligned}
$$




$$
\begin{aligned}
& \underline{\beta}_{D}(w, s) \stackrel{\text { def }}{=}\left[\delta_{\{i=j\}} \frac{1-\alpha_{j}(s+\rho(j) w)}{s+\rho(j) w}\right], \\
& \zeta_{i j}(w, s) \stackrel{\text { def }}{=} \prod_{n=i}^{j} \alpha_{n}(s+\rho(n) w) \beta_{n}(w) ; \zeta_{i j}(w, s)=1 \text { for } i>j \\
& \underline{\underline{\alpha}}^{*}(w, s) \stackrel{\text { def }}{=}\left[\begin{array}{ccccc}
0 & \zeta_{11}(w, s) & 0 & \ldots & 0 \\
0 & 0 & \zeta_{22}(w, s) & \ldots & 0 \\
\vdots & \ldots & \ldots & \ldots & \zeta_{J-1, J-1}(w, s) \\
0 & \ldots & \ldots & \ldots & 0
\end{array}\right] \\
& \underline{\underline{\alpha}}_{D}^{*}(w, s) \stackrel{\text { def }}{=}\left[\begin{array}{cccc}
\zeta_{1 J}(w, s) & & \underline{0} \\
& \zeta_{2 J}(w, s) & & \\
\underline{\underline{0}} & & \ddots & \\
& & & \zeta_{J J}(w, s)
\end{array}\right] \text {. }
\end{aligned}
$$

Here, it should be noted that the diagonal components of $\underline{D}_{D}(w, s)$ in (3.12) are the Laplace transforms of the survival function $\bar{A}_{j}(x)$ from the fact that

$$
\frac{1-\alpha_{j}(s+\rho(j) w)}{s+\rho(j) w}=\int_{0}^{\infty} e^{-\{s+\rho(j) w\} x} \bar{A}_{j}(x) d x
$$

We also define the following matrices.

$$
\underline{\underline{1}} \stackrel{\text { def }}{=}\left[\begin{array}{ccc}
1 & \cdots & 1 \\
\vdots & \cdots & \vdots \\
1 & \cdots & 1
\end{array}\right], \underline{\underline{I}} \stackrel{\text { def }}{=}\left[\begin{array}{lll}
1 & & \underline{\underline{0}} \\
& \ddots & \\
\underline{\underline{0}} & & 1
\end{array}\right] .
$$

A few preliminary lemmas are needed.

Lemma 3.1 For $\underline{\underline{\hat{\xi}}}_{k}(0+, w, s)$ defined in (3.11), one has

$$
\underline{\underline{\hat{\xi}}}_{k}(0+, w, s)=4 \begin{array}{ll}
{\left[\underline{\underline{I}}-\underline{\underline{\alpha}}^{*}(w, s)\right]^{-1},} & k=0 \\
\left\{\zeta_{1 J}(w, s)\right\}^{k} \underline{\underline{\alpha}}_{D}^{*}(w, s) \underline{\underline{1}} \underline{\underline{\alpha}}_{D}^{*-1}(w, s), & k \geqslant 1
\end{array} .
$$

\section{Proof}

Substituting (3.3) into (3.5), it can be seen that

$$
\begin{aligned}
& f_{0: i j}(0+, z, t) \\
= & \delta_{\{j=i\}} \delta(z) \delta(t)+\delta_{\{j>i\}} \int_{0}^{\infty} \int_{-\infty}^{\infty}\left\{\delta_{\{j-1=i\}} \delta\left(z-z^{\prime}-\rho(j-1) t\right) \delta(t-x) \bar{A}_{j-1}(x)\right. \\
+ & \left.\delta_{\{j-1>i\}} f_{0: i, j-1}\left(0+, z-z^{\prime}-\rho(j-1) x, t-x\right) \bar{A}_{j-1}(x)\right\} \eta_{j-1}(x) b_{j-1}\left(z^{\prime}\right) d z^{\prime} d x .(3.1
\end{aligned}
$$

Similarly, substitution of (3.4) into (3.6) yields

$$
= \begin{cases}\left\{\begin{array}{l}
k: i j \\
\int_{0}^{\infty}(0+, z, t)
\end{array} \int_{-\infty}^{\infty} f_{k-1: i J}\left(0+, z-z^{\prime}-\rho(J) x, t-x\right) a_{J}(x) b_{J}\left(z^{\prime}\right) d z^{\prime} d x,\right. & j=1 \\
\int_{0}^{\infty} \int_{-\infty}^{\infty} f_{k: i, j-1}\left(0+, z-z^{\prime}-\rho(j-1) x, t-x\right) a_{j-1}(x) b_{j-1}\left(z^{\prime}\right) d z^{\prime} d x, & 2 \leqslant j \leqslant J\end{cases}
$$


By taking Laplace transforms with respect to $t$ in (3.18) and (3.19), it then follows that

$$
\begin{aligned}
& \hat{\xi}_{0: i j}(0+, z, s) \\
= & \delta_{\{j=i\}} \delta(z)+\delta_{\{j>i\}} \int_{0}^{\infty} e^{-s x}\left[\int _ { - \infty } ^ { \infty } \left\{\delta_{\{j-1=i\}} \delta\left(z-z^{\prime}-\rho(j-1) x\right)\right.\right. \\
+ & \left.\left.\delta_{\{j-1>i\}} \hat{\xi}_{0: i, j-1}\left(0+, z-z^{\prime}-\rho(j-1) x, s\right)\right\} a_{j-1}(x) b_{j-1}\left(z^{\prime}\right) d z^{\prime}\right] d x,
\end{aligned}
$$

and

$$
\begin{aligned}
& \oint_{k: i j}(0+, z, s) \\
& =\begin{array}{ll}
\int_{0}^{\infty} \int_{-\infty}^{\infty} e^{-s x} \hat{\xi}_{k-1: i J}\left(0+, z-z^{\prime}-\rho(J) x, s\right) a_{J}(x) b_{J}\left(z^{\prime}\right) d z^{\prime} d x, & j=1 \\
\int_{0}^{\infty} \int_{-\infty}^{\infty} e^{-s x} \hat{\xi}_{k: i, j-1}\left(0+, z-z^{\prime}-\rho(j-1) x, s\right) a_{j-1}(x) b_{j-1}\left(z^{\prime}\right) d z^{\prime} d x, & 2 \leqslant j \leqslant J
\end{array} .
\end{aligned}
$$

If we again take Laplace-Fourier transforms with respect to $z$ in (3.20) and (3.21), one has and

$$
\begin{aligned}
& \hat{\hat{\xi}}_{0: i j}(0+, w, s)=\delta_{\{j=i\}}+\left\{\delta_{\{j-1=i\}}+\delta_{\{j-1>i\}} \hat{\hat{\xi}}_{0: i, j-1}(0+, w, s)\right\} \zeta_{j-1, j-1}(w, s) \\
& \hat{\hat{\xi}}_{k: i j}(0+, w, s)=\begin{array}{ll}
\hat{\hat{\xi}}_{k-1: i J}(0+, w, s) \zeta_{J J}(w, s), & j=1 \\
\hat{\hat{\xi}}_{k: i, j-1}(0+, w, s) \zeta_{j-1, j-1}(w, s), & 2 \leqslant j \leqslant J
\end{array}
\end{aligned}
$$

since $\zeta_{j j}(w, s)=\alpha_{j}(s+\rho(j) w) \beta_{j}(w)$.

Equations in (3.22) and (3.23) can be rewritten in matrix form using $\underline{\underline{\hat{\xi}}}_{k}(0+, w, s)$ defined in (3.11) in the following manner. We first note that

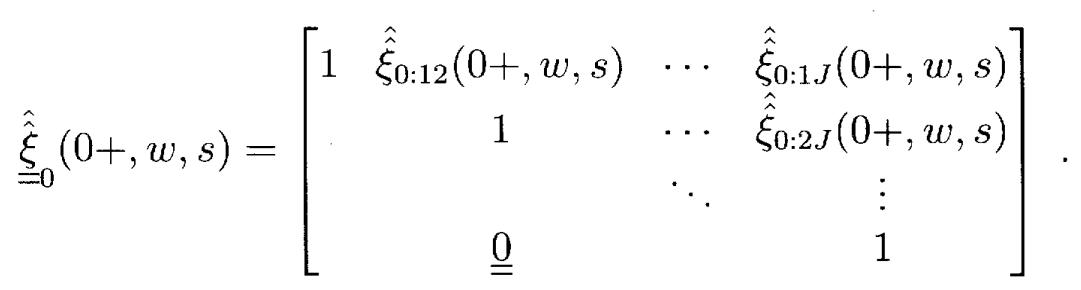

From (3.22), this then leads to

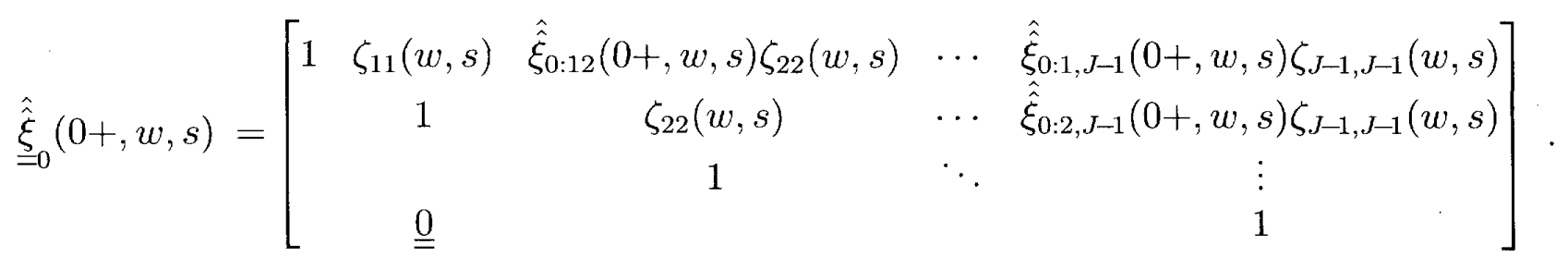

The matrix on the right hand side of (3.25) can be rewritten from $(3.14)$ as $\underline{\underline{I}}+\underline{\hat{\hat{\xi}}}_{0}(0+, w, s) \underline{\underline{\alpha}}^{*}(w, s)$, so that

$$
\underline{\hat{\xi}}_{0}(0+, w, s)=\left[\underline{\underline{I}}-\underline{\underline{\alpha}}^{*}(w, s)\right]^{-1}=\left[\begin{array}{ccccc}
1 & \zeta_{11}(w, s) & \zeta_{12}(w, s) & \cdots & \zeta_{1, J-1}(w, s) \\
& 1 & \zeta_{22}(w, s) & \cdots & \zeta_{2, J-1}(w, s) \\
& & 1 & \cdots & \zeta_{3, J-1}(w, s) \\
& \underline{0} & & \ddots & \vdots \\
& & & & 1
\end{array}\right]
$$


proving the case for $k=0$.

For $k \geqslant 1$, we prove by induction. When $k=1$, one sees that

$$
\begin{aligned}
& \hat{\hat{\xi}}_{1}(0+, w, s) \\
= & {\left[\begin{array}{ccc}
\hat{\hat{\xi}}_{1: 11}(0+, w, s) & \cdots & \hat{\hat{\xi}}_{1: 1 J}(0+, w, s) \\
\vdots & \ldots & \vdots \\
\hat{\hat{\xi}}_{1: J 1}(0+, w, s) & \cdots & \hat{\hat{\xi}}_{1: J J}(0+, w, s)
\end{array}\right] } \\
= & {\left[\begin{array}{cccc}
\hat{\hat{\xi}}_{0: 1 J}(0+, w, s) \zeta_{J J}(w, s) & \hat{\hat{\xi}}_{1: 11}(0+, w, s) \zeta_{11}(w, s) & \cdots & \hat{\hat{\xi}}_{1: 1, J-1}(0+, w, s) \zeta_{J-1, J-1}(w, s) \\
\vdots & \vdots & \cdots & \vdots \\
\hat{\hat{\xi}}_{0: J J}(0+, w, s) \zeta_{J J}(w, s) & \hat{\hat{\xi}}_{1: J 1}(0+, w, s) \zeta_{11}(w, s) & \cdots & \hat{\hat{\xi}}_{1: J, J-1}(0+, w, s) \zeta_{J-1, J-1}(w, s)
\end{array}\right] . }
\end{aligned}
$$

By employing (3.22) and (3.23) in the above expression, it follows that

$$
\underline{\hat{\xi}}_{1}(0+, w, s)=\left[\begin{array}{cccc}
\zeta_{1 J}(w, s) & \zeta_{1 J}(w, s) \zeta_{11}(w, s) & \cdots & \zeta_{1 J}(w, s) \zeta_{1, J-1}(w, s) \\
\zeta_{2 J}(w, s) & \zeta_{2 J}(w, s) \zeta_{11}(w, s) & \cdots & \zeta_{2 J}(w, s) \zeta_{1, J-1}(w, s) \\
\vdots & \vdots & \cdots & \vdots \\
\zeta_{J J}(w, s) & \zeta_{J J}(w, s) \zeta_{11}(w, s) & \cdots & \zeta_{J J}(w, s) \zeta_{1, J-1}(w, s)
\end{array}\right]
$$

where the last column results from (3.13), (3.22) and (3.23). From (3.15) and (3.16), this then leads to

$$
\underline{\underline{\hat{\xi}}}_{1}(0+, w, s)=\underline{\underline{\alpha}}_{D}^{*}(w, s) \underline{\underline{1}}\left[\begin{array}{cccc}
1 & & & \underline{0} \\
& \zeta_{11}(w, s) & & \\
& \ddots & \\
& \underline{\underline{0}} & & \zeta_{1, J-1}(w, s)
\end{array}\right] .
$$

It should be noted from (3.13) and (3.15) that

$$
\underline{\underline{\alpha}}_{D}^{*}(w, s)\left[\begin{array}{cccc}
1 & & & \underline{\underline{0}} \\
& \zeta_{11}(w, s) & & \\
& \underline{\underline{0}} & & \zeta_{1, J-1}(w, s)
\end{array}\right]=\zeta_{1 J}(w, s) \underline{\underline{I}}
$$

so that one has

$$
\left[\begin{array}{cccc}
1 & & & \underline{\underline{0}} \\
& \zeta_{11}(w, s) & & \\
& & \ddots & \\
& \underline{\underline{0}} & & \zeta_{1, J-1}(w, s)
\end{array}\right]=\zeta_{1 J}(w, s) \underline{\underline{\alpha}}_{D}^{*-1}(w, s)
$$

where $\underline{\underline{\alpha}}_{D}^{*-1}(w, s)$ is given from $(3.15)$ as

$$
\underline{\underline{\alpha}}_{D}^{*-1}(w, s)=\left[\begin{array}{cccc}
\frac{1}{\zeta_{1 J}(w, s)} & & & \underline{\underline{0}} \\
& \frac{1}{\zeta_{2 J}(w, s)} & & \\
& & \ddots & \\
\underline{\underline{0}} & & & \frac{1}{\zeta_{J J}(w, s)}
\end{array}\right] .
$$


Substituting (3.28) into (3.27), one concludes that

$$
\underline{\underline{\hat{\xi}}}_{1}(0+, w, s)=\zeta_{1 J}(w, s) \underline{\underline{\alpha}}_{D}^{*}(w, s) \underline{\underline{1}}_{D}^{*-1}(w, s)
$$

completing the proof for $k=1$.

Suppose the statement holds true for $k-1$ and consider the case for $k$. It can be seen from (3.23) that

$$
\begin{aligned}
& \hat{\hat{\xi}}_{k}(0+, w, s) \\
= & {\left[\begin{array}{ccc}
\hat{\hat{\xi}}_{k: 11}(0+, w, s) & \cdots & \hat{\hat{\xi}}_{k: 1 J}(0+, w, s) \\
\hat{\hat{\xi}}_{k: 21}(0+, w, s) & \cdots & \hat{\hat{\xi}}_{k: 2 J}(0+, w, s) \\
\vdots & \vdots & \vdots \\
\hat{\hat{\xi}}_{k: J 1}(0+, w, s) & \cdots & \hat{\hat{\xi}}_{k: J J}(0+, w, s)
\end{array}\right] } \\
= & {\left[\begin{array}{cccc}
\hat{\hat{\xi}}_{k-1: 1 J}(0+, w, s) \zeta_{J J}(w, s) & \hat{\hat{\xi}}_{k: 11}(0+, w, s) \zeta_{11}(w, s) & \cdots & \hat{\hat{\xi}}_{k: 1, J-1}(0+, w, s) \zeta_{J-1, J-1}(w, s) \\
\hat{\hat{\xi}}_{k-1: 2 J}(0+, w, s) \zeta_{J J}(w, s) & \hat{\hat{\xi}}_{k: 21}(0+, w, s) \zeta_{11}(w, s) & \cdots & \hat{\xi}_{k: 2, J-1}(0+, w, s) \zeta_{J-1, J-1}(w, s) \\
\vdots & \cdots & \cdots & \vdots \\
\hat{\hat{\xi}}_{k-1: J J}(0+, w, s) \zeta_{J J}(w, s) & \hat{\hat{\xi}}_{k: J 1}(0+, w, s) \zeta_{11}(w, s) & \cdots & \hat{\hat{\xi}}_{k: J, J-1}(0+, w, s) \zeta_{J-1, J-1}(w, s)
\end{array}\right] . }
\end{aligned}
$$

The last matrix in the above expression can be rewritten in matrix product form as

$$
\begin{aligned}
\underline{\hat{\xi}}_{k}(0+, w, s)= & {\left[\begin{array}{cccc}
\hat{\hat{\xi}}_{k-1: 1 J}(0+, w, s) & \hat{\hat{\xi}}_{k: 11}(0+, w, s) & \cdots & \hat{\hat{\xi}}_{k: 1, J-1}(0+, w, s) \\
\hat{\hat{\xi}}_{k-1: 2 J}(0+, w, s) & \hat{\hat{\xi}}_{k: 21}(0+, w, s) & \cdots & \hat{\hat{\xi}}_{k: 2, J-1}(0+, w, s) \\
\vdots & \ldots & \vdots & \vdots \\
\hat{\hat{\xi}}_{k-1: J J}(0+, w, s) & \hat{\hat{\xi}}_{k: J 1}(0+, w, s) & \cdots & \hat{\hat{\xi}}_{k: J, J-1}(0+, w, s)
\end{array}\right] } \\
& {\left[\begin{array}{cccc}
\zeta_{J J}(w, s) & & \underline{\underline{0}} \\
& \zeta_{11}(w, s) & & \\
\underline{\underline{0}} & & \ddots & \\
& & & \zeta_{J-1, J-1}(w, s)
\end{array}\right] . }
\end{aligned}
$$

By applying (3.23) to the first matrix in the above expression, one sees that

$$
\begin{aligned}
& \underline{\hat{\xi}}_{k}(0+, w, s) \\
& =\left[\begin{array}{ccccc}
\hat{\hat{\xi}}_{k-1: 1, J-1}(0+, w, s) & \hat{\hat{\xi}}_{k-1: 1 J}(0+, w, s) & \hat{\hat{\xi}}_{k: 11}(0+, w, s) & \cdots & \hat{\hat{\xi}}_{k: 1, J-2}(0+, w, s) \\
\hat{\hat{\xi}}_{k-1: 2, J-1}(0+, w, s) & \hat{\hat{\xi}}_{k-1: 2 J}(0+, w, s) & \hat{\hat{\xi}}_{k: 12}(0+, w, s) & \cdots & \hat{\hat{\xi}}_{k: 2, J-2}(0+, w, s) \\
\vdots & \ldots & \vdots & \vdots & \vdots \\
\hat{\hat{\xi}}_{k-1: J, J-1}(0+, w, s) & \hat{\hat{\xi}}_{k-1: J J}(0+, w, s) & \hat{\hat{\xi}}_{k: 1 J}(0+, w, s) & \cdots & \hat{\hat{\xi}}_{k: J, J-2}(0+, w, s)
\end{array}\right] \\
& \cdot\left[\begin{array}{cccc}
\zeta_{J-1, J-1}(w, s) & & & \underline{\underline{0}} \\
& \zeta_{J J}(w, s) & & \\
\underline{\underline{0}} & & \ddots & \\
& & & \zeta_{J-2, J-2}(w, s)
\end{array}\right] \cdot\left[\begin{array}{cccc}
\zeta_{J J}(w, s) & & & \underline{\underline{0}} \\
& \zeta_{11}(w, s) & & \\
& & \ddots & \\
\underline{\underline{0}} & & & \zeta_{J-1, J-1}(w, s)
\end{array}\right]
\end{aligned}
$$


By repeating this procedure, it follows that

$$
\underline{\underline{\hat{\xi}}}_{k}(0+, w, s)=\zeta_{1 J}(w, s) \underline{\underline{\hat{\xi}}}_{k-1}(0+, w, s),
$$

where $\zeta_{1 J}(w, s)=\prod_{j=1}^{J} \zeta_{j j}(w, s)$ is employed from (3.13). The lemma now follows from the induction hypothesis.

Lemma 3.2 For the multivariate process $[N(t), J(t), X(t), Z(t)]$ with $N(0)=X(0)=$ $Z(0)=0$ and $J(0)=i$, let $\underline{\underline{\hat{\varphi}}}_{k}(v, w, s)$ be defined as in (3.9). Then

$$
\underline{\hat{\hat{\varphi}}}_{k}(v, w, s)=\left\{\begin{array}{ll}
{\left[\underline{\underline{I}}-\underline{\underline{\alpha}}^{*}(w, s)\right]^{-1} \underline{\underline{\beta}}_{D}(w, v+s),} & k=0 \\
\left\{\zeta_{1 J}(w, s)\right\}^{k} \underline{\underline{\alpha}}_{D}^{*}(w, s) \underline{\underline{1}}_{D}^{*-1}(w, s) \underline{\underline{\beta}}_{D}(w, v+s), & k \geqslant 1
\end{array} .\right.
$$

\section{Proof}

By taking Laplace transforms of (3.3) and (3.4) with respect to $t$, one sees that

$$
\hat{\varphi}_{k: i j}(x, z, s)=\left\{\begin{array}{ll}
\delta_{\{j=i\}} \delta(z-\rho(j) x) e^{-s x} \bar{A}_{j}(x) & \\
+\delta_{\{j>i\}} e^{-s x} \hat{\xi}_{0: i j}(0+, z-\rho(j) x, s) \bar{A}_{j}(x), & k=0 . \\
e^{-s x} \hat{\xi}_{k: i j}(0+, z-\rho(j) x, s) \bar{A}_{j}(x), & k \geqslant 1
\end{array} .\right.
$$

If Laplace-Fourier transforms are taken again with respect to $z$ in (3.33), one has

$$
\hat{\hat{\varphi}}_{k: i j}(x, w, s)=\left\{\begin{array}{ll}
{\left[\delta_{\{j=i\}}+\delta_{\{j>i\}} \hat{\hat{\xi}}_{0: i j}(0+, w, s)\right] e^{-(s+\rho(j) w) x} \bar{A}_{j}(x),} & k=0 \\
\hat{\xi}_{k: i j}(0+, w, s) e^{-(s+\rho(j) w) x} \bar{A}_{j}(x), & k \geqslant 1
\end{array} .\right.
$$

By taking Laplace transforms one more time with respect to $x$ in (3.34), it follows that

$$
\hat{\hat{\hat{\varphi}}}_{k: i j}(v, w, s)=\left\{\begin{array}{ll}
{\left[\delta_{\{j=i\}}+\delta_{\{j>i\}} \hat{\hat{\xi}}_{0: i j}(0+, w, s)\right] \frac{1-\alpha_{j}(v+\rho(j) w+s)}{v+\rho(j) w+s},} & k=0 \\
\hat{\hat{\xi}}_{k: i j}(0+, w, s) \cdot \frac{1-\alpha_{j}(v+\rho(j) w+s)}{v+\rho(j) w+s}, & k \geqslant 1
\end{array} .\right.
$$

Noting (3.24) for $k=0$, the above two cases can be combined for $k \geqslant 0$ in matrix form as

$$
\underline{\underline{\hat{\varphi}}}_{k}(v, w, s)=\underline{\hat{\hat{\xi}}}_{k}(0+, w, s) \underline{\underline{\beta}}_{D}(w, v+s), k \geqslant 0 \text {. }
$$

Substituting (3.17) of Lemma 3.1 into (3.36), the lemma follows.

Lemma 3.3 Let

$$
\underline{\underline{\chi}}(w, s, u) \stackrel{\text { def }}{=}[\underline{\underline{I}}-\underline{\underline{\zeta}}(w, s, u)]^{-1}
$$

where

$$
\underline{\zeta}(w, s, u) \stackrel{\operatorname{def}}{=}\left[\begin{array}{ccccc}
0 & \zeta_{11}(w, s) & 0 & \cdots & 0 \\
0 & 0 & \zeta_{22}(w, s) & \cdots & 0 \\
0 & 0 & \cdots & \ddots & \vdots \\
0 & 0 & \cdots & 0 & \zeta_{J-1, J-1}(w, s) \\
u \zeta_{J J}(w, s) & 0 & \cdots & 0 & 0
\end{array}\right] .
$$

Then one has

$$
\underline{\underline{\chi}}(w, s, u)=\left[\underline{\underline{I}}-\underline{\underline{\alpha}}^{*}(w, s)\right]^{-1}+\frac{u \zeta_{1 J}(w, s)}{1-u \zeta_{1 J}(w, s)} \underline{\underline{\alpha}}_{D}^{*}(w, s) \underline{\underline{1}}_{\underline{\alpha}_{D}^{*-1}}(w, s) .
$$




\section{Proof}

From (3.15), (3.16) and (3.29), one finds that

$$
\underline{\underline{\alpha}}_{D}^{*}(w, s) \underline{\underline{1}} \underline{\underline{\alpha}}_{D}^{*-1}(w, s)=\left[\begin{array}{cccc}
1 & \frac{\zeta_{1 J}(w, s)}{\zeta_{2 J}(w, s)} & \cdots & \frac{\zeta_{1 J}(w, s)}{\zeta_{J J}(w, s)} \\
\frac{\zeta_{2 J}(w, s)}{\zeta_{1 J}(w, s)} & 1 & \cdots & \frac{\zeta_{2 J}(w, s)}{\zeta_{J J}(w, s)} \\
\vdots & \vdots & \ddots & \vdots \\
\frac{\zeta_{J J}(w, s)}{\zeta_{1 J}(w, s)} & \frac{\zeta_{J J}(w, s)}{\zeta_{2 J}(w, s)} & \cdots & 1
\end{array}\right] .
$$

For $m, n \in \mathcal{J}$, it should be noted from (3.13) that

$$
\frac{\zeta_{m J}(w, s)}{\zeta_{n J}(w, s)}= \begin{cases}\zeta_{m, n-1}(w, s) & \text { if } m<n \\ 1 & \text { if } m=n \\ \frac{1}{\zeta_{n, m-1}(w, s)} & \text { if } m>n\end{cases}
$$

Substituting (3.41) into (3.40) then yields

$$
\underline{\underline{\alpha}}_{D}^{*}(w, s) \underline{\underline{1}} \underline{\underline{\alpha}}_{D}^{*-1}(w, s)=\left[\begin{array}{cccc}
1 & \zeta_{11}(w, s) & \cdots & \zeta_{1, J-1}(w, s) \\
\frac{1}{\zeta_{11}(w, s)} & 1 & \cdots & \zeta_{2, J-1}(w, s) \\
\vdots & \vdots & \ddots & \vdots \\
\frac{1}{\zeta_{1, J-1}(w, s)} & \frac{1}{\zeta_{2, J-1}(w, s)} & \cdots & 1
\end{array}\right] .
$$

From (3.42) and (3.26), it can be seen that

$$
\begin{aligned}
& {\left[\underline{\underline{I}}-\underline{\underline{\alpha}}^{*}(w, s)\right]^{-1}+\frac{u \zeta_{1 J}(w, s)}{1-u \zeta_{1 J}(w, s)} \underline{\underline{\alpha}}_{D}^{*}(w, s) \underline{\underline{1}} \underline{\underline{\alpha}}_{D}^{*-1}(w, s)=} \\
& \frac{1}{1-u \zeta_{1 J}(w, s)}\left[\begin{array}{ccccc}
1 & \zeta_{11}(w, s) & \zeta_{12}(w, s) & \cdots & \zeta_{1, J-1}(w, s) \\
u \zeta_{2 J}(w, s) & 1 & \zeta_{22}(w, s) & \cdots & \zeta_{2, J-1}(w, s) \\
u \zeta_{3 J}(w, s) & u \zeta_{11}(w, s) \zeta_{3 J}(w, s) & 1 & \cdots & \zeta_{3, J-1}(w, s) \\
\vdots & \vdots & \vdots & \ddots & \vdots \\
u \zeta_{J J}(w, s) & u \zeta_{11}(w, s) \zeta_{J J}(w, s) & u \zeta_{12}(w, s) \zeta_{J J}(w, s) & \cdots & 1
\end{array}\right] .
\end{aligned}
$$

By multiplying $\underline{\underline{\chi}}^{-1}(w, s, u)$ from the right in (3.43), one concludes that

$$
\left[\left[\underline{\underline{I}}-\underline{\underline{\alpha}}^{*}(w, s)\right]^{-1}+\frac{u \zeta_{1 J}(w, s)}{1-u \zeta_{1 J}(w, s)} \underline{\underline{\alpha}}_{D}^{*}(w, s) \underline{\underline{1}} \underline{\underline{\alpha}}_{D}^{*-1}(w, s)\right] \underline{\underline{\chi}}^{-1}(w, s, u)=\underline{\underline{I}},
$$

completing the proof.

By taking the generating function of $\underline{\hat{\hat{\varphi}}}_{k}(v, w, s)$ in $(3.32)$ with respect to $k(k=0,1,2 \cdots)$, the joint transform of $[N(t), J(t), X(t), Z(t)]$ can be obtained.

Theorem 3.4 Let $\underline{\underline{\hat{\hat{\varphi}}}}(v, w, s, u)$ be the matrix generating function of $\underline{\hat{\hat{\varphi}}}_{k}(v, w, s)$ in (3.32) defined by

$$
\underline{\hat{\hat{\varphi}}}(v, w, s, u) \stackrel{\text { def }}{=}\left[\hat{\hat{\hat{\varphi}}}_{i j}(v, w, s, u)\right] ; \hat{\hat{\hat{\varphi}}}_{i j}(v, w, s, u) \stackrel{\text { def }}{=} \sum_{k=0}^{\infty} \hat{\hat{\hat{\varphi}}}_{k: i j}(v, w, s) u^{k}
$$

Then one has

$$
\underline{\underline{\hat{\varphi}}}(v, w, s, u)=\underline{\underline{\chi}}(w, s, u) \underline{\underline{\beta}}_{D}(w, v+s),
$$

where $\underline{\underline{\beta}}_{D}(w, v+s)$ is as given in (3.12). 


\section{Proof}

Multiplying $u^{k}$ to both sides of (3.32) and then summing from $k=0$ to $\infty$, one finds that

$$
\begin{aligned}
& \underline{\hat{\hat{\varphi}}}(v, w, s, u)=\left[\underline{\underline{I}}-\underline{\underline{\alpha}}^{*}(w, s)\right]^{-1} \underline{\underline{\beta}}_{D}(w, v+s) \\
& +u \zeta_{1 J}(w, s) \sum_{k=1}^{\infty}\left\{u \zeta_{1 J}(w, s)\right\}^{k-1} \underline{\underline{\alpha}}_{D}^{*}(w, s) \underline{\underline{1}}_{D}^{*-1}(w, s) \underline{\underline{\beta}}_{D}(w, v+s)
\end{aligned}
$$

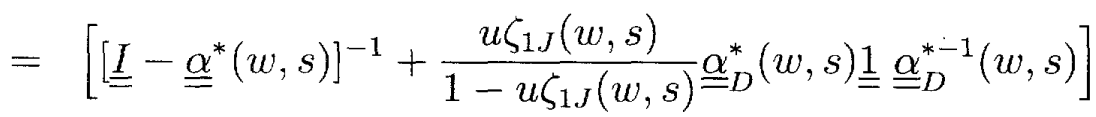

$$
\begin{aligned}
& \text { - } \underline{\beta}_{D}(w, v+s) \text {. }
\end{aligned}
$$

Substituting (3.39) into (3.46) then yields (3.45), completing the proof.

Remark 3.5 By setting $u=1$ in (3.45), Theorem 3.4 is reduced to a special case of Theorem 2.8.1 of Masuda [17] and (2.3) of Masuda [18]. Indeed, $\underline{\zeta}(w, s, 1)$ is the bivariate transform of $J(t)$ and $Z(t)$, where $\underline{\underline{\alpha}}^{* *}(w, s)$ of Masuda [17] and the matrix $\left[Q_{i j}^{*}(w, s)\right]$ of Masuda [18] are equal to $\underline{\underline{\zeta}}(w, s, 1)$.

\section{Asymptotic Expansion of $\mathbf{E}[Z(t)]$ and $\operatorname{Cor}[N(t), Z(t)]$}

The purpose of this section is to establish the asymptotic expansions of $\mathrm{E}[Z(t)]$ and $\operatorname{Cor}[N(t), Z(t)]$ as $t \rightarrow \infty$. In order to accomplish this, we introduce Theorem 1 of Keilson [12]. Let $A_{j: k}$ be the $k$-th moment of $X_{j}$. More formally, we define

$$
A_{j: k} \stackrel{\text { def }}{=} \int_{0}^{\infty} x^{k} a_{j}(x) d x
$$

The following matrix is also employed.

$$
\underline{A}_{k} \stackrel{\text { def }}{=}\left[\begin{array}{ccccc}
0 & A_{1: k} & 0 & \cdots & 0 \\
0 & 0 & A_{2: k} & \cdots & 0 \\
0 & 0 & \cdots & \ddots & \vdots \\
0 & 0 & \cdots & 0 & A_{J-1: k} \\
A_{J: k} & 0 & \cdots & 0 & 0
\end{array}\right] .
$$

If $\int_{0}^{\infty} x^{2} d A_{j}(x)<0$ for all $j$ and $A_{j}(x)$ are not lattice distribution with a common span, one has

$$
\underline{\underline{\chi}}(0, s, 1)=\frac{1}{s} \underline{\underline{H}}_{1}+\underline{\underline{H}}_{0}+\underline{\underline{o}}(1)
$$

as $s \rightarrow 0+$, where, for $\underline{e}_{d}^{\top}=\underline{e}_{d}^{\top} \underline{\underline{A}}_{0}$, the two matrices $\underline{\underline{H}}_{0}$ and $\underline{\underline{H}} 1$ are given as

$$
\underline{\underline{H}}_{1} \stackrel{\text { def }}{=} \frac{1}{m_{1}} \underline{J} ; \stackrel{J}{=} \stackrel{\text { def }}{=} \underline{1} \underline{e}_{d}^{\top} ; m_{1} \stackrel{\text { def }}{=} \underline{e}_{d}^{\top} \stackrel{A}{\underline{A}}_{1} \underline{1}
$$

and

$$
\underline{\underline{H}}_{0} \stackrel{\text { def }}{=} \underline{\underline{H}}_{1}\left(-\underline{\underline{A}}_{1}+\frac{1}{2} \underline{\underline{A}}_{2} \underline{\underline{H}}_{1}\right)+\left(\underline{\underline{Z}}-\underline{\underline{H}}_{1} \underline{\underline{A}}_{1} \underline{\underline{Z}}\right)\left(\underline{\underline{A}}_{0}-\underline{\underline{A}}_{1} \underline{\underline{H}}_{1}\right)+\underline{\underline{I}}
$$


Here, $\underline{\underline{Z}}$ is the fundamental matrix associated with the Markov chain governed by $\underline{A}_{0}$, i.e.

$$
\underline{\underline{Z}} \stackrel{\text { def }}{=}\left[\underline{\underline{I}}-\underline{\underline{A}}_{0}+\underline{\underline{J}}\right]^{-1}
$$

Using Lemmas A.1 and A.2 in Appendix, the following theorem holds.

Theorem 4.1 For the matrices in Lemmas $A .1$ and A.2, let

$$
\left.\underline{\underline{X}}_{1} \stackrel{\text { def }}{=} \underline{\underline{H}}_{1} \underline{\underline{A}}_{D: 1} \underline{\underline{\rho}}^{\#}+\underline{\underline{D}}_{1}^{\#}\right) \underline{\underline{H}}_{1} \underline{\underline{A}}_{D: 1} \text { and } \underline{\underline{X}}_{0} \stackrel{\text { def }}{=} \frac{1}{2} \underline{\underline{V}}_{2} \underline{\underline{A}}_{D: 2}-\underline{\underline{V}}_{1} \underline{\underline{A}}_{D: 1}+\frac{1}{2} \underline{\underline{H}}_{1} \underline{\underline{\rho}}_{D} \underline{\underline{A}}_{D: 2} .
$$

a) Ast $\rightarrow \infty$, one has

$$
\begin{aligned}
\underline{E}[Z(t) \mid J(0)=i] & \stackrel{\text { def }}{=}[E[Z(t), J(t)=1 \mid J(0)=i] \cdots E[Z(t), J(t)=J \mid J(0)=i]]^{\top} \\
& =\underline{u}_{i}^{\top}\left(\underline{\underline{X}}_{1} t+\underline{\underline{X}}_{0}\right)+\underline{o}(1)
\end{aligned}
$$

where $\underline{u}_{i}$ is the $i$-th unit vector.

b) Given the initial probability vector $\underline{p}^{\top}(0)$ of $J(t)$, one has

$$
E[Z(t)]=\underline{p}^{\top}(0)\left(\underline{\underline{X}}_{1} t+\underline{\underline{X}}_{0}\right) \underline{1}+o(1)
$$

\section{Proof}

Setting $v=0$ and $u=1$ in (3.45) leads to $\underline{\underline{\hat{\varphi}}}(0, w, s, 1)=\underline{\underline{\chi}}(w, s, 1) \underline{\underline{\beta}}_{D}(w, s)$. By differentiating this expression with respect to $w$ and setting $w=0$, it can be seen that

$$
\left.\frac{\partial}{\partial w}\{\underline{\underline{\hat{\hat{\varphi}}}}(0, w, s, 1)\}\right|_{w=0}=\left.\left\{\frac{\partial}{\partial w} \underline{\underline{\chi}}(w, s, 1)\right\}\right|_{w=0} \underline{\underline{\beta}}_{D}(0, s)+\left.\underline{\underline{\chi}}(0, s, 1)\left\{\frac{\partial}{\partial w} \underline{\beta}_{D}(w, s)\right\}\right|_{w=0} .
$$

Applying (4.3), Lemmas A.1 c), d) and A.2 e) to (4.8), it then follows that

$$
\begin{aligned}
\left.\frac{\partial}{\partial w}\{\underline{\underline{\hat{\varphi}}}(0, w, s, 1)\}\right|_{w=0} & =\left\{\frac{1}{s^{2}} \underline{\underline{V}}_{2}+\frac{1}{s} \underline{\underline{V}}_{1}+\underline{\underline{o}}\left(\frac{1}{s}\right)\right\}\left\{\underline{\underline{A}}_{D: 1}-\frac{1}{2} s \underline{\underline{A}}_{D: 2}+\underline{\underline{o}}(s)\right\} \\
& +\left\{\frac{1}{s} \underline{\underline{H}}_{1}+\underline{\underline{H}}_{0}+\underline{\underline{o}}(1)\right\}\left\{-\frac{1}{2} \underline{\underline{\rho}} \underline{\underline{A}}_{D: 2}+\underline{\underline{o}}(1)\right\} \\
& =-\left(\frac{1}{s^{2}} \underline{\underline{X}}_{1}+\frac{1}{s} \underline{\underline{X}}_{0}\right)+\underline{o}\left(\frac{1}{s}\right) .
\end{aligned}
$$

Hence one has

$$
\begin{aligned}
\underline{E}[Z(t) \mid J(0)=i] & =-\underline{u}_{i}^{\top} \mathcal{L}^{-1}\left\{\left.\frac{\partial}{\partial w} \hat{\hat{\hat{\varphi}}}(0, w, s, 1)\right|_{w=0}\right\} \\
& =\underline{u}_{i}^{\top}\left(\underline{\underline{X}}_{1} t+\underline{\underline{X}}_{0}\right)+\underline{o}(1) \text { as } t \rightarrow \infty
\end{aligned}
$$

where $\mathcal{L}^{-1}$ means the inversion of the Laplace transform, i.e., $\mathcal{L}^{-1}\{\alpha(s)\}=a(t)$ with $\alpha(s)=$ $\mathcal{L}\{a(t)\}=\int_{0}^{\infty} e^{-s t} a(t) d t$. Part $\left.b\right)$ is immediate from Part $a$ ), proving the theorem.

We next turn our attention to the asymptotic expansion of Cor $[N(t), Z(t)]$. 
Theorem 4.2 Ast $\rightarrow \infty$, one has

$$
\operatorname{Cor}[N(t), Z(t)]=\frac{C o_{2} t^{2}+C o_{1} t+o(t)}{\sqrt{n_{2} z_{2} t^{4}+\left(n_{2} z_{1}+n_{1} z_{2}\right) t^{3}+n_{1} z_{1} t^{2}+o\left(t^{2}\right)}}
$$

where

$$
\begin{aligned}
& n_{2} \stackrel{\text { def }}{=} \underline{p}^{\top}(0) \underline{S}_{2} \underline{1}-\left(\underline{p}^{\top}(0) \underline{\underline{L}}_{1} \underline{1}\right)^{2} ; n_{1} \stackrel{\text { def }}{=} \underline{p}^{\top}(0) \underline{\underline{S}}_{1} \underline{1}-2 \underline{p}^{\top}(0) \underline{\underline{L}}_{1} \underline{1}^{\top}(0) \underline{\underline{L}}_{0} \underline{1} ; \\
& z_{2} \stackrel{\text { def }}{=} \underline{p}^{\top}(0) \underline{\underline{T}}_{2} \underline{1}-\left(\underline{p}^{\top}(0) \underline{\underline{X}}_{1} \underline{1}\right)^{2} ; z_{1} \stackrel{\text { def }}{=} \underline{p}^{\top}(0) \underline{\underline{T}}_{1} \underline{1}-2 \underline{p}^{\top}(0) \underline{\underline{X}}_{1} \underline{\underline{1}} \underline{p}^{\top}(0) \underline{\underline{X}}_{0} \underline{1} ; \\
& C o_{2} \stackrel{\text { def }}{=} \underline{p}^{\top}(0) \underline{\underline{U}}_{2} \underline{1}-\underline{p}^{\top}(0) \underline{\underline{L}}_{1} \underline{1} \underline{p}^{\top}(0) \underline{\underline{X}}_{1} \underline{1} ; \text { and } \\
& C o_{1} \stackrel{\text { def }}{=} \underline{p}^{\top}(0) \underline{\underline{U}}_{1} 1-\underline{p}^{\top}(0) \underline{\underline{L}}_{1} \underline{\underline{1}} \underline{\underline{p}}^{\top}(0) \underline{\underline{X}}_{0} \underline{1}-\underline{p}^{\top}(0) \underline{\underline{L}}_{0} \underline{\underline{p}}^{\top}(0) \underline{\underline{X}}_{1} \underline{1} .
\end{aligned}
$$

\section{Proof}

By differentiating $\underline{\hat{\hat{\varphi}}}(0, w, s, u)$ with respect to $u$ and $w$, and letting $u=1$ and $w=0$, one sees that

$$
\mathrm{E}[N(t) Z(t)]=-\underline{p}^{\top}(0) \mathcal{L}^{-1}\left\{\left.\frac{\partial^{2}}{\partial u \partial w} \underline{\hat{\hat{\varphi}}}(0, w, s, u)\right|_{u=1, w=0}\right\} \underline{1}
$$

The asymptotic expansion of the above expression is given in Lemma A.3 $d$ ), which in turn yields that of

$$
\operatorname{Cov}[N(t), Z(t)]=\mathrm{E}[N(t) Z(t)]-\mathrm{E}[N(t)] \mathrm{E}[Z(t)]
$$

More specifically, using Theorem $4.1 \mathrm{~b}$ ), Lemma A.3 a) and $d$ ), one finds that

$$
\operatorname{Cov}[N(t), Z(t)]=C o_{2} t^{2}+C o_{1} t+o(t)
$$

One also sees from Lemma A.3 that

$$
\begin{aligned}
& \mathrm{V}[N(t)]=n_{2} t^{2}+n_{1} t+o(t) \\
& \mathrm{V}[Z(t)]=z_{2} t^{2}+z_{1} t+o(t)
\end{aligned}
$$

The theorem then follows from (4.10), (4.11) and (4.12) since Cor $[N(t), Z(t)]=\operatorname{Cov}[N(t), Z(t)] /$ $\sqrt{\mathrm{V}[N(t)] \mathrm{V}[Z(t)]}$.

In the next section, we investigate the optimal preventive maintenance policy for the production system discussed in Section 1 based on Theorem $4.1 \mathrm{~b}$ ). In order to highlight the importance of the dynamic analysis of the cyclic renewal process, a simplified classical renewal model is introduced. Although the long-term optimal policy for the cyclic renewal model coincides with that for the simplified classical renewal model, dynamic optimal policies of the two models could significantly differ from each other when the planning horizon $T$ is not sufficiently large. This point will be illustrated numerically in Section 6 . 


\section{Long-term and Dynamic Optimal Preventive Maintenance Policies for Pro- duction Management}

Within the context of the preventive maintenance model discussed in Section 1, we now consider the problem of determining when to conduct a complete overhaul so as to maximize the reward rate per unit time in the time interval $(0, T]$. This means that $J$, representing the number of minimal repairs before the complete overhaul, should be determined optimally. Since $\lim _{T \rightarrow \infty} \mathrm{E}[Z(T)] / T=\underline{p}^{\top}(0) \underline{\underline{X}}_{1} \underline{1}$ from Theorem $4.1 \mathrm{~b}$ ), given the initial probability vector $\underline{p}^{\top}(0)$, let the long-term optimal policy $J^{*}$ be defined by

$$
J^{*} \stackrel{\text { def }}{=} \arg \max _{J}\left\{\underline{p}^{\top}(0) \underline{\underline{X}}_{1} \underline{1}\right\}
$$

Exploiting the second dominating term as $T \rightarrow \infty$ in Theorem $4.1 \mathrm{~b}$ ), the dynamic optimal policy $J^{* *}$ can be defined similarly as

$$
J^{* *} \stackrel{\text { def }}{=} \arg \max _{J}\left\{\underline{p}^{\top}(0)\left(\underline{\underline{X}}_{1}+\frac{\underline{X}_{0}}{T}\right) \underline{1}\right\}
$$

As far as the long-term optimal policy is concerned, one may work with the following simplified model. Let $\left\{N_{\operatorname{Ren}}(T) ; T \geqslant 0\right\}$ be a renewal process associated with a sequence of i.i.d. random variables $Y_{i}$ where $Y_{i}$ are as specified in (2.2). Namely, one has $N_{\text {Ren }}(T)=n$ if and only if $S_{n} \leqslant T<S_{n+1}$, where $S_{n} \stackrel{\text { def }}{=} \sum_{i=1}^{n} Y_{i}$. The classical renewal theory, see e.g. Cox [4], states that the asymptotic expansion of $\mathrm{E}\left[N_{\operatorname{Ren}}(T)\right]$ is given by

$$
\mathrm{E}\left[N_{\operatorname{Ren}}(T)\right]=\frac{T}{\widetilde{A}_{1}}+\frac{\widetilde{A}_{2}-2 \widetilde{A}_{1}^{2}}{2 \widetilde{A}_{1}^{2}}+o(1)
$$

where $\widetilde{A}_{k} \stackrel{\text { def }}{=} \sum_{j=1}^{J} A_{j: k}$ for $k=1,2$ with $A_{j: k}$ as defined in (4.1). Let $W_{i}$ be the reward within the $i$-th renewal period. The simplified reward process $Z_{\text {Simp }}(T)$ may then be defined as

$$
Z_{\text {Simp }}(T) \stackrel{\text { def }}{=} \sum_{i=1}^{N_{R e n}(T)} W_{i}
$$

Since $W_{i}$ are i.i.d. and independent of $N_{R e n}(T)$ with

$$
\mathrm{E}[W]=\sum_{j=1}^{J}\left\{\mathrm{E}\left[D_{j}\right]+\rho(j) A_{j: 1}\right\}
$$

the asymptotic expansion of $\mathrm{E}\left[Z_{\text {Simp }}(T)\right]$ is obtained from (5.3) and (5.4) as

$$
\mathrm{E}\left[Z_{S i m p}(T)\right]=\mathrm{E}\left[N_{R e n}(T)\right] \mathrm{E}[W]=\frac{\mathrm{E}[W]}{\widetilde{A}_{1}} T+\frac{\mathrm{E}[W]}{2 \widetilde{A}_{1}^{2}}\left(\widetilde{A}_{2}-2 \widetilde{A}_{1}^{2}\right)+o(1) .
$$

Assuming that $J(0)=1$ and $J(T)=j<J$, the simplified reward process $Z_{\text {Simp }}(T)$ differs from the original process $Z(T)$ in that

$$
\begin{aligned}
\mathrm{E}\left[Z_{\text {Simp }}(T) \mid J(T)=j\right] & =\mathrm{E}[Z(T) \mid J(T)=j]+\rho(j) \mathrm{E}\left[X_{\text {Res:j }}(T)\right] \\
& +\sum_{m=j+1}^{J} \rho(m) \mathrm{E}\left[X_{m}\right]+\sum_{m=j}^{J} \mathrm{E}\left[D_{m}\right]
\end{aligned}
$$


where $X_{\text {Res:j }}(T)$ is the residual dwell time of $J(T)$ in state $j$ at time $T$.

From (5.7), the expected reward rate per unit time under $Z_{\text {Simp }}(T)$ and that under $Z(T)$ should coincide each other as $T \rightarrow \infty$. Consequently, the long-term optimal policy under $Z_{\text {Simp }}(T)$ should be the same as that under $Z(T)$. When the planning horizon $T$ is not sufficiently large, however, the optimal policy under $Z_{S i m p}(T)$ may be quite different from that under $Z(T)$, as we will see in the next section. In order to highlight these points, in parallel with (5.1) and (5.2), we define $J_{\text {Simp }}^{*}$ and $J_{\text {Simp }}^{* *}$ as

$$
J_{S i m p}^{*} \stackrel{\text { def }}{=} \arg \max _{J}\left\{\frac{\mathrm{E}[W]}{\widetilde{A}_{1}}\right\}
$$

and

$$
J_{\text {Simp }}^{* *} \stackrel{\text { def }}{=} \arg \max _{J}\left\{\frac{\mathrm{E}[W]}{\widetilde{A_{1}}}+\frac{\mathrm{E}[W]}{2 \widetilde{A}_{1}^{2} T}\left(\widetilde{A}_{2}-2 \widetilde{A}_{1}^{2}\right)\right\} .
$$

Theorem 5.1 Let $J^{*}$ and $J_{\text {Simp }}^{*}$ be as in (5.1) and (5.8) respectively. One then has $J^{*}=J_{\text {Simp }}^{*}$

\section{Proof}

From (4.2), $\underline{\underline{A}}_{0}$ is a permutation matrix and therefore doubly stochastic. Consequently $\underline{e}_{d}^{\top}$ satisfying $\underline{e}_{d}^{\top}=\underline{e}_{d}^{\top} \underline{A}_{0}$ is given as $\underline{e}_{d}^{\top}=\underline{1}^{\top}$. It follows from (4.4) that $m_{1}=\widetilde{A}_{1}, \underline{\underline{J}}=\underline{\underline{1}}$ and $\underline{\underline{H}}_{1}=\frac{1}{\widetilde{A}_{1}} \underline{\underline{1}}$. For $\underline{\underline{X}}_{1}$ given in Theorem 4.1 together with $\mathrm{E}[W]$ in (5.5), these observations then lead to

$$
\begin{aligned}
\underline{p}^{\top}(0) \underline{\underline{X}}_{1} \underline{1} & =\underline{p}^{\top}(0)\left\{\frac{1}{\widetilde{A}_{1}} \underline{\underline{1}}\left(\underline{\underline{A}}_{D: 1} \underline{\underline{\rho}}^{\#}+\underline{\underline{D}}_{1}^{\#}\right) \cdot \frac{1}{\widetilde{A}_{1}} \underline{\underline{1}}_{\underline{\underline{A}}}^{\underline{\underline{A}}}{ }_{D: 1}\right\} \underline{1} \\
& =\frac{1}{\widetilde{A}_{1}^{2}}\left[A_{J: 1} \rho(J)+\mathrm{E}\left[D_{J}\right], A_{1: 1} \rho(1)+\mathrm{E}\left[D_{1}\right], \cdots, A_{J-1: 1} \rho(J-1)+\mathrm{E}\left[D_{J-1}\right]\right] \cdot \widetilde{A}_{1} \underline{1} \\
& =\frac{\mathrm{E}[W]}{\widetilde{A}_{1}},
\end{aligned}
$$

and the theorem follows from (5.1) and (5.8).

\section{Danger of Exclusive Reliance on Long-term Analysis: Numerical Results}

We consider a production system where the system down cost is huge. A typical example may be the production of semi-conductor chips because the production machines are extremely expensive and the repair takes a long time since vendor engineers often have to be called in once the system fails. In such a situation, preventive maintenance is widely practiced where minimal repairs take place as minor problems occur, which can be addressed by on-site engineers. A complete overhaul demanding the presence of vendor engineers is conducted only after minimal repairs are repeated certain many times, as depicted in Figure 6.1. Here, we note that $J=2 K+2$ and $X_{2}, X_{4}, \cdots, X_{2 K}$ represent minimal repairs while $X_{2 K+2}$ describes the time required for conducting a complete overhaul. The question then is to determine when to conduct a complete overhaul. The reward process defined on the cyclic renewal process proposed in this paper provides a useful computational vehicle for numerically exploring optimal preventive maintenance policies of this sort in a dynamic environment. In this section, we demonstrate this claim using Theorem $4.1 \mathrm{~b}$ ). 


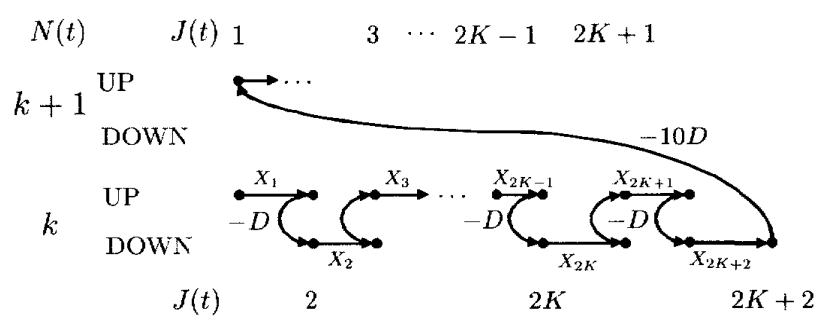

Figure 6.1: Typical sample path of $[N(t), J(t)]$ for preventive maintenance model

The idea behind minimal repairs is to prolong the availability of the system in the time interval $(0, T]$ by accommodating a partial system adjustment from time to time. This approach can be effective since minimal repairs can be done at much lower cost and in much shorter time in comparison with a complete overhaul. Starting with a fresh system lifetime, it is natural to assume that the time until the next minimal repair becomes shorter while the subsequent minimal repair time becomes longer as this alternating process is repeated. When it is decided to conduct a complete overhaul, the system is brought back to its original fresh state upon completion of the overhaul.

In order to incorporate this probabilistic structure, we employ Gamma variates. More specifically, let $\left\{\widehat{X}_{i}\right\}_{i=1}^{\infty}$ and $\left\{\widetilde{X}_{i}\right\}_{i=1}^{\infty}$ be sequences of i.i.d. exponential random variables with parameters $\lambda$ and $\mu$ respectively, where the former is used to construct system lifetimes while the latter is employed to structure repair times. The system lifetime $X_{1}$ when it is in the fresh state is assumed to be a Gamma variate of integral order $K(0)$ with scaling parameter $\lambda$, i.e.,

$$
X_{1}=\sum_{i=1}^{K(0)} \widehat{X}_{i}
$$

We also assume that the time required for conducting a complete overhaul is a Gamma variate of integral order $K(1)$ with scaling parameter $\mu$. Assuming that $K$ minimal repairs would take place, one has

$$
X_{2(K+1)}=\sum_{i=1}^{K(1)} \widetilde{X}_{i}
$$

So as to reflect the fact that the time until the next minimal repair becomes shorter while the subsequent ninimal repair time becomes longer as this cycle is repeated, we define

$$
X_{j}=4 \begin{array}{ll}
\widehat{X}_{1}+\widehat{X}_{2}+\cdots+\widehat{X}_{K(2)+2-(j+1) / 2} & \text { if } j=3,5, \cdots, 2 K+1 \\
\widetilde{X}_{1}+\widetilde{X}_{2}+\cdots+\widetilde{X}_{j / 2} & \text { if } j=2,4, \cdots, 2 K
\end{array}
$$

where $K(2)$ is a parameter satisfying $K \leqslant K(2) \leqslant K(0)$. For $j$ odd, $X_{j}$ is the time until the next minimal repair which decreases stochastically with respect to $j$. For $j$ even, $X_{j}$ is the subsequent minor repair time which increases stochastically in $j$.

Let $\alpha_{j}(s)$ be the Laplace transform of the p.d.f of $X_{j}$. From (6.1), (6.2) and (6.3), it can 
be seen that

$$
\alpha_{j}(s)=\left\{\begin{array}{ll}
\left(\frac{\lambda}{s+\lambda}\right)^{K(0)} & \text { if } j=1 \\
\left(\frac{\mu}{s+\mu}\right)^{K(1)} & \text { if } j=2(K+1) \\
\left(\frac{\lambda}{s+\lambda}\right)^{K(2)+2-\frac{j+1}{2}} & \text { if } j=3,5, \cdots, 2 K+1 \\
\left(\frac{\mu}{s+\mu}\right)^{\frac{j}{2}} & \text { if } j=2,4, \cdots, 2 K
\end{array} .\right.
$$

By differentiating (6.4) with respect to $s$ once or twice and setting $s=0$, one finds that

$$
\mathrm{E}\left[X_{j}\right]= \begin{cases}\frac{K(0)}{\lambda} & \text { if } j=1 \\ \frac{K(1)}{\mu} & \text { if } j=2(K+1) \\ \frac{1}{\lambda}\left(K(2)+2-\frac{j+1}{2}\right) & \text { if } j=3,5, \cdots, 2 K+1 \\ \frac{j}{2 \mu} & \text { if } j=2,4, \cdots, 2 K\end{cases}
$$

and

$$
\mathrm{E}\left[X_{j}^{2}\right]=\left\{\begin{array}{ll}
\frac{1}{\lambda} K(0)(K(0)+1) & \text { if } j=1 \\
\frac{1}{\mu} K(1)(K(1)+1) & \text { if } j=2(K+1) \\
\frac{1}{\lambda}\left(K(2)+2-\frac{j+1}{2}\right)\left(K(2)+3-\frac{j+1}{2}\right) & \text { if } j=3,5, \cdots, 2 K+1 \\
\frac{j}{2 \mu}\left(\frac{j}{2}+1\right) & \text { if } j=2,4, \cdots, 2 K
\end{array} .\right.
$$

We next turn our attentign to the reward structure. The reward rate function $\rho(j)$ is defined as

$$
\rho(j)=4 \begin{array}{ll}
\rho_{\mathrm{UP}} & \text { if } j=1,3, \cdots, 2 K+1 \\
-\rho_{\text {DOWN }} & \text { if } j=2,4, \cdots, 2 K+2
\end{array},
$$

where $\rho_{\mathrm{UP}}$ and $\rho_{\text {DOWN }}$ are parameters satisfying $\rho_{\mathrm{UP}}>0$ and $\rho_{\text {DOWN }}>0$. The fixed cost for calling in on-site engineers for a minimal repair and that for calling in vendor engineers for a complete overhaul can be expressed in terms of random reward jumps $D_{j}$. The associated means are defined as

$$
\mathrm{E}\left[D_{j}\right]=\left\{\begin{array}{ll}
-D & \text { if } j=1,3, \cdots, 2 K+1 \\
0 & \text { if } j=2,4, \cdots, 2 K \\
-10 D & \text { if } j=2 K+2
\end{array} .\right.
$$

In what follows, a set of parameter values for $\lambda, \rho_{\text {Down }}, D, i, K(0), K(1)$ and $K(2)$ as well as $\underline{p}^{\top}(0)$ would be fixed as specified in Table 6.1 below. In order to demonstrate the danger of exclusive reliance on the long-term reward rate, we first define 
Table 6.1: Parameter values for $\lambda, \rho_{\text {Down }}, D, i, K(0), K(1), K(2)$ and $\underline{p}^{\top}(0)$

\begin{tabular}{|c|c|c|c|c|c|c|c|}
\hline $\bar{\lambda}$ & $\rho_{\text {DOWN }}$ & $D$ & $i$ & $K(0)$ & $K(1)$ & $K(2)$ & $p^{\top}(0)$ \\
\hline 3 & 10 & 100 & 1 & 100 & 100 & 50 & {$\left[\begin{array}{llll}1 & \overline{0} & \cdots & 0\end{array}\right]$} \\
\hline
\end{tabular}

where the long-term rate $L T R$ and the dynamic rate $D R(T)$ are given from Theorem 4.1 b) as

$$
L T R=\underline{p}^{\top}(0) \underline{\underline{X}}_{1} \underline{1} ; D R(T)=\frac{\underline{p}^{\top}(0) \underline{\underline{X}}_{0} \underline{1}}{T}
$$

Figure 6.2 illustrates that the convergence of $D R(T)$ to 0 as $T \rightarrow \infty$ is not necessarily fast and therefore it is dangerous to exclusively rely upon $L T R$ to devise the optimal preventive maintenance policy when the planning horizon $T$ is not sufficiently large.
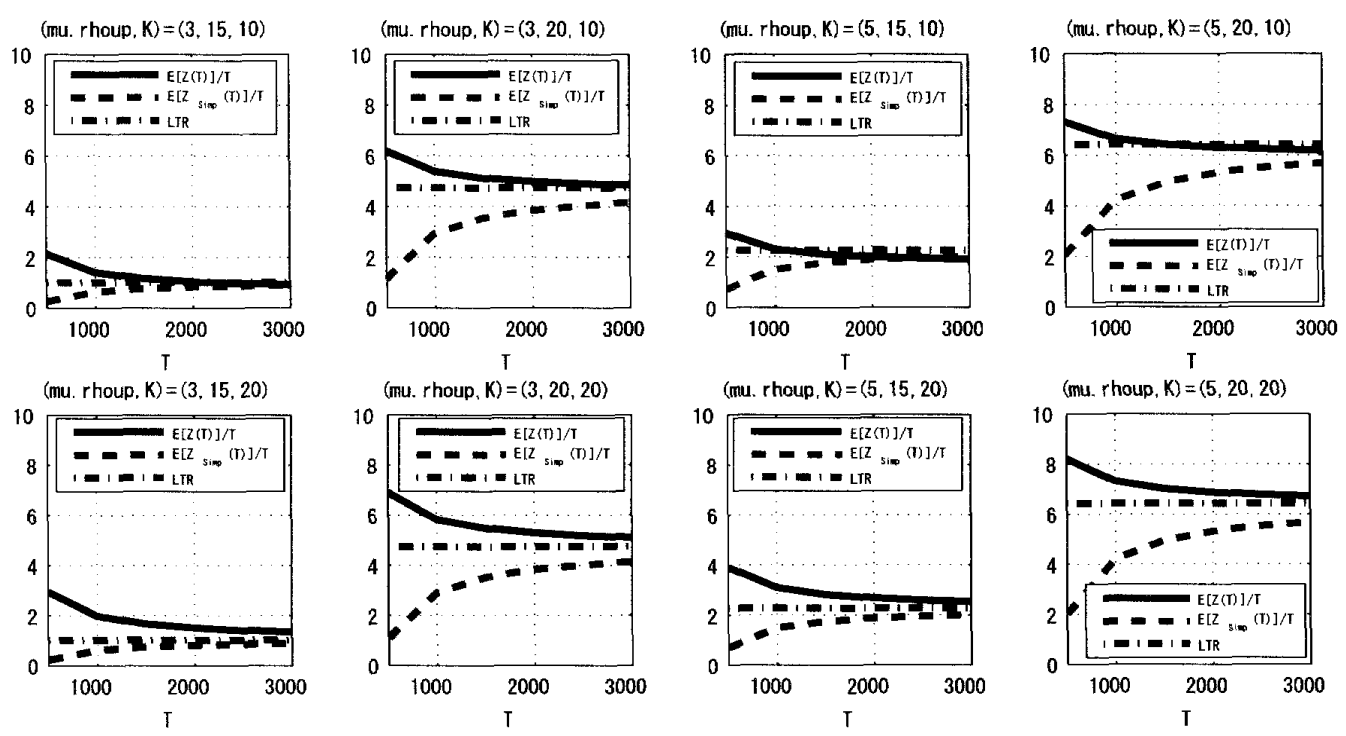

Figure 6.2: $\mathrm{E}[Z(T)] / T, \mathrm{E}\left[Z_{\text {Simp }}(T)\right] / T$ and $L T R$ for $\mu=3,5, \rho_{\mathrm{UP}}=15,20$ and $K=10,20$

The above danger can be observed more directly by exploring the impact of $D R(T)$ on the optimal preventive maintenance policies. For this purpose, corresponding to (5.1), (5.2), (5.8) and (5.9), we define

$$
\begin{aligned}
K^{*} & \stackrel{\text { def }}{=} \arg \max _{K}\{L T R\} \\
K_{T}^{* *} & \stackrel{\text { def }}{=} \arg \max _{K}\{L T R+D R(T)\} \\
K_{\text {Simp }}^{*} & \stackrel{\text { def }}{=} \arg \max _{K}\left\{\frac{\mathrm{E}[W]}{\widetilde{A}_{1}}\right\}, \\
K_{T: S i m p}^{* *} & \stackrel{\text { def }}{=} \arg \max _{K} \mathrm{E}\left[Z_{\text {Simp }}(T)\right] / T=\arg \max _{K}\left\{\frac{\mathrm{E}[W]}{\widetilde{A}_{1}}+\frac{\mathrm{E}[W]}{2 \widetilde{A}_{1}^{2} T}\left(\widetilde{A}_{2}-2 \widetilde{A}_{1}^{2}\right)\right\},
\end{aligned}
$$

where it should be noted from Theorem 5.1 that $K^{*}=K_{\text {Simp }}^{*}$. Numerical experiments are conducted to explore $K^{*}, K_{T}^{* *}$ and $K_{T: S i m p}^{* *}$ as $\mu, \rho_{\mathrm{UP}}$ and $T$ are varied, where the results 
are summarized in Table 6.2 . It can be seen that $K_{T}^{* *}$ could be quite different from $K^{*}$ and $K_{T: \text { Simp }}^{* *}$ when $T$ is small and $\mu$ or $\rho_{\mathrm{UP}}$ are large, demonstrating the necessity of the detailed analysis of the cyclic renewal process under such parameter values.

Table 6.2: $K_{T}^{* *}, K_{T: S i m p}^{* *}$ and $K^{*}$ for each pair of $\mu=3,5,15$ and $\rho_{\mathrm{UP}}=15,20$ for $T=$ $500,1000, \cdots, 3000\left(K_{T}^{* *} \backslash K_{T: S i m p}^{* *}\right)$

\begin{tabular}{cccccccc}
\hline$\left(\mu, \rho_{\mathrm{UP}}\right) \backslash T$ & 500 & 1000 & 1500 & 2000 & 2500 & 3000 & $K^{*}=K_{\text {Simp }}^{*}$ \\
\hline$(3,15)$ & $18 \backslash 13$ & $17 \backslash 15$ & $16 \backslash 15$ & $16 \backslash 15$ & $16 \backslash 15$ & $16 \backslash 15$ & 16 \\
\hline$(3,20)$ & $17 \backslash 10$ & $16 \backslash 12$ & $15 \backslash 13$ & $15 \backslash 13$ & $15 \backslash 14$ & $15 \backslash 14$ & 15 \\
\hline$(5,15)$ & $19 \backslash 12$ & $18 \backslash 15$ & $17 \backslash 15$ & $17 \backslash 16$ & $17 \backslash 16$ & $17 \backslash 16$ & 17 \\
\hline$(5,20)$ & $18 \backslash 10$ & $17 \backslash 12$ & $17 \backslash 13$ & $16 \backslash 14$ & $16 \backslash 14$ & $16 \backslash 15$ & 16 \\
\hline$(15,15)$ & $22 \backslash 12$ & $20 \backslash 15$ & $20 \backslash 17$ & $19 \backslash 17$ & $19 \backslash 17$ & $19 \backslash 18$ & 19 \\
\hline$(15,20)$ & $21 \backslash 9$ & $19 \backslash 13$ & $19 \backslash 14$ & $19 \backslash 15$ & $19 \backslash 14$ & $19 \backslash 16$ & 18 \\
\hline
\end{tabular}

We next turn our attention to $\operatorname{Cor}[N(T), Z(T)]$ for capturing the time-dependent correlation structure numerically based on Theorem 4.2. Parameter values for $\lambda, \rho_{\mathrm{DOWN}}, D, i$, $K(0), K(1)$ and $K(2)$ are again as in Table 6.1. Figure 6.3 illustrates $\operatorname{Cor}[N(T), Z(T)]$ for each pair of $\mu=3,5,15$ and $\rho_{\mathrm{UP}}=15,20$ as functions of $K$. For these parameter values, one finds that $\operatorname{Cor}[N(T), Z(T)]$ is unimodal with respect to $K$. When $\rho_{\text {UP }}=20$, it can be seen that $\operatorname{Cor}[N(T), Z(T)]$ increases as $\mu$ increases. For $T=500$ and $\rho_{\mathrm{UP}}=20, \operatorname{Cor}[N(T), Z(T)]$ is monotonically increasing as a function of $K$ for all values of $\mu=3,5,15$.
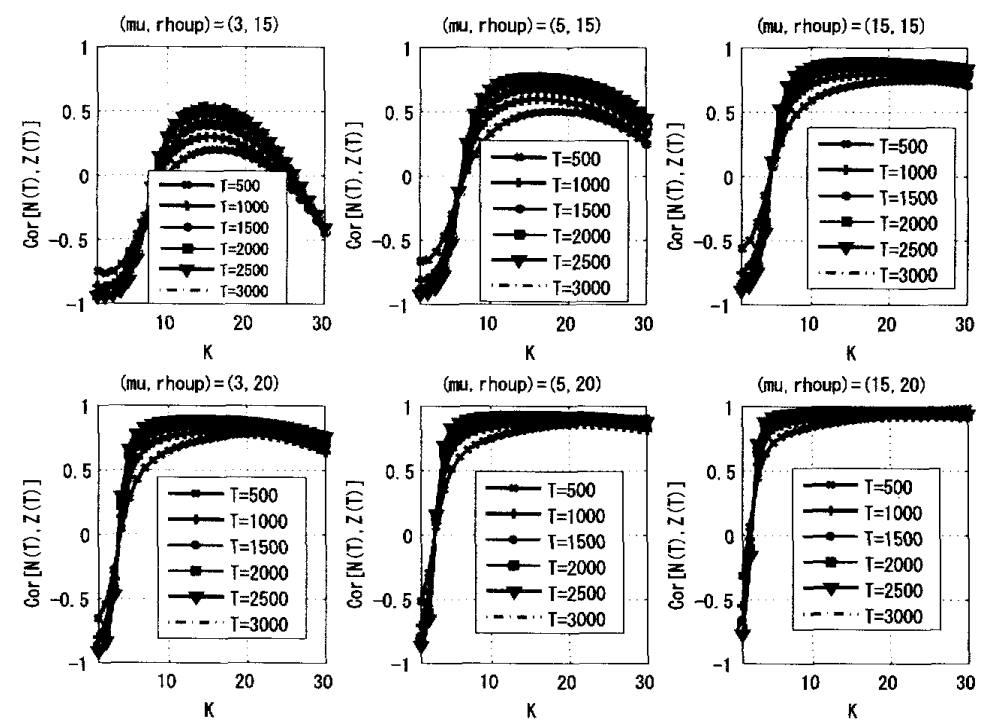

Figure 6.3: $\operatorname{Cor}[N(T), Z(T)]$ for each pair of $\mu=3,5,15$ and $\rho_{\mathrm{UP}}=15,20$ for $T=500$, $1000, \cdots, 3000$

\section{Acknowledgement}

The authors wish to thank two anonymous referees for many helpful comments. In particular, Section 5 is newly written in response to them, improving the earlier version of this paper substantially. This research is supported by MEXT Grant-in-Aid for Scientific Research (C) 17510114. 


\section{Appendix}

In this appendix, we establish various lemmas concerning the asymptotic expansions of the transform results obtained in Section 3. These lemmas can be proven from (4.3) combined with appropriate differentiation in a straightforward manner, and the proofs are omitted. The asymptotic theorems needed for numerically exploring the underlying reward and correlation structure are derived in Section 4 using these lemmas.

Let $\underline{\underline{\alpha}}^{\#}(s)$ and $\underline{\underline{1}}^{\#}$ be the matrices defined by

$$
\underline{\underline{\alpha}}^{\#}(s) \stackrel{\text { def }}{=}\left[\begin{array}{ccccc}
0 & \alpha_{1}(s) & 0 & \cdots & 0 \\
0 & 0 & \alpha_{2}(s) & \cdots & 0 \\
0 & 0 & \cdots & \ddots & \vdots \\
0 & 0 & \cdots & 0 & \alpha_{J-1}(s) \\
\alpha_{J}(s) & 0 & \cdots & 0 & 0
\end{array}\right], \underline{1} \stackrel{\#}{=} \stackrel{\text { def }}{=}\left[\begin{array}{ccccc}
0 & 1 & 0 & \cdots & 0 \\
0 & 0 & 1 & \cdots & 0 \\
0 & 0 & \cdots & \ddots & \vdots \\
0 & 0 & \cdots & 0 & 1 \\
1 & 0 & \cdots & 0 & 0
\end{array}\right] .
$$

Lemma A.1 For $A_{j: k}$ defined in (4.1), we define $\underline{\underline{A}}_{D: k} \stackrel{\text { def }}{=} \operatorname{diag}\left[A_{j: k}\right]$ and $\stackrel{\rho}{=} \stackrel{\text { def }}{=} \operatorname{diag}[\rho(j)]$ $(j=1, \cdots, J, k=1,2)$. As $s \rightarrow 0+$, the following expressions hold true.
a) $\underline{\underline{\alpha}}^{\#}(s)=\underline{\underline{1}}^{\#}-s \underline{\underline{A}}_{1}+\frac{1}{2} s^{2} \underline{\underline{A}}_{2}+\underline{\underline{o}}\left(s^{2}\right)$
b) $\frac{d}{d s} \underline{\underline{\alpha}}^{\#}(s)=-\underline{\underline{A}}_{1}+s \underline{\underline{A}}_{2}+\underline{\underline{o}}(s) ;\left(\frac{d}{d s}\right)^{2} \underline{\underline{\alpha}}^{\#}(s)=\underline{\underline{A}}_{2}+\underline{\underline{o}}(1)$
c) $\underline{\underline{\beta}}_{D}(0, s)=\underline{\underline{A}}_{D: 1}-\frac{1}{2} s \underline{\underline{A}}_{D: 2}+\underline{\underline{o}}(s)$
d) $\left.\frac{\partial}{\partial w} \underline{\underline{\beta}}(w, s)\right|_{w=0}=-\frac{1}{2} \underline{\underline{\rho}}{ }_{D} \underline{\underline{A}}_{D: 2}+\underline{\underline{o}}(1) ;\left.\frac{\partial^{2}}{\partial w^{2}} \underline{\underline{\beta}}_{D}(w, s)\right|_{w=0}=\underline{\underline{o}}\left(\frac{1}{s}\right)$

Lemma A.2 As $s \rightarrow 0+$, the following statements hold.
a) $\left.\left\{\frac{\partial}{\partial u} \underline{\underline{\chi}}(0, s, u)\right\}\right|_{u=1}=\frac{1}{s^{2}} \underline{\underline{Q}} 2+\frac{1}{s} \underline{\underline{Q}} 1+\underline{\underline{o}}\left(\frac{1}{s}\right)$
b) $\left.\left\{\frac{\partial^{2}}{\partial u^{2}} \underline{\underline{\chi}}(0, s, u)\right\}\right|_{u=1}=\frac{1}{s^{3}} \underline{\underline{K}}_{3}+\frac{1}{s^{2}} \underline{\underline{K}}_{2}+\underline{\underline{o}}\left(\frac{1}{s^{2}}\right)$
c) $\left.\left\{\frac{\partial}{\partial w} \underline{\underline{ }}(w, s, 1)\right\}\right|_{w=0}=-\left(\underline{\underline{A}}_{D: 1} \underline{\underline{\rho}}^{\#}+\underline{\underline{D}}_{1}^{\#}\right)+s\left(\underline{\underline{A}}_{D: 2} \underline{\underline{\rho}}^{\#}+\underline{\underline{A}}_{D: 1} \underline{\underline{D}}_{1}^{\#}\right)+\underline{\underline{o}}(s)$
d) $\left.\left\{\frac{\partial^{2}}{\partial w^{2}} \underline{\underline{\zeta}}(w, s, 1)\right\}\right|_{w=0}=\underline{\underline{A}}_{D: 2} \underline{\underline{\rho}}_{D} \underline{\underline{\rho}}^{\#}+2 \underline{\underline{A}}_{D: 1} \underline{\underline{\rho}}_{D} \underline{\underline{D}}_{1}^{\#}+\underline{\underline{D}}_{2}^{\#}+\underline{\underline{o}}(1)$
e) $\left.\left\{\frac{\partial}{\partial w} \underline{\underline{\chi}}(w, s, 1)\right\}\right|_{w=0}=\frac{1}{s^{2}} \underline{\underline{V}}_{2}+\frac{1}{s} \underline{\underline{V}}_{1}+\underline{\underline{o}}\left(\frac{1}{s}\right)$
f) $\left.\left\{\frac{\partial^{2}}{\partial w^{2}} \underline{\underline{\chi}}(w, s, 1)\right\}\right|_{w=0}=\frac{1}{s^{3}} \underline{\underline{W}}_{3}+\frac{1}{s^{2}} \underline{\underline{W}}_{2}+\underline{\underline{o}}\left(\frac{1}{s^{2}}\right)$
g) $\left.\left\{\frac{\partial^{2}}{\partial u \partial w} \underline{\underline{\chi}}(w, s, u)\right\}\right|_{u=1, w=0}=\frac{1}{s^{3}} \underline{\underline{R}}_{3}+\frac{1}{s^{2}} \underline{\underline{R}}_{2}+\underline{\underline{o}}\left(\frac{1}{s^{2}}\right)$ 
where

$$
\begin{aligned}
& \stackrel{\rho}{=} \stackrel{\text { def }}{=}\left[\begin{array}{ccccc}
0 & \rho(1) & 0 & \cdots & 0 \\
0 & 0 & \rho(2) & \cdots & 0 \\
0 & 0 & \cdots & \ddots & \vdots \\
0 & 0 & \cdots & 0 & \rho(J-1) \\
\rho(J) & 0 & \cdots & 0 & 0
\end{array}\right] \\
& \underline{D}_{1}^{\# \stackrel{\text { def }}{=}}\left[\begin{array}{ccccc}
0 & E\left[D_{1}\right] & 0 & \cdots & 0 \\
0 & 0 & E\left[D_{2}\right] & \cdots & 0 \\
0 & 0 & \cdots & \ddots & \vdots \\
0 & 0 & \cdots & 0 & E\left[D_{J-1}\right] \\
E\left[D_{J}\right] & 0 & \cdots & 0 & 0
\end{array}\right] \\
& \underline{\underline{D}}_{2}^{\#} \stackrel{\text { def }}{=}\left[\begin{array}{ccccc}
0 & E\left[D_{1}^{2}\right] & 0 & \cdots & 0 \\
0 & 0 & E\left[D_{2}^{2}\right] & \cdots & 0 \\
0 & 0 & \cdots & \ddots & \vdots \\
0 & 0 & \cdots & 0 & E\left[D_{J-1}^{2}\right] \\
E\left[D_{J}^{2}\right] & 0 & \cdots & 0 & 0
\end{array}\right], \underline{\underline{1}} \stackrel{\text { def }}{=}\left[\begin{array}{cc}
0 & \\
\vdots & \underline{0} \\
0 & \\
1 &
\end{array}\right] \text {, } \\
& \underline{\underline{Q}}_{2} \stackrel{\text { def }}{=} \underline{\underline{H}}_{1} \underline{\underline{\hat{1}}} \underline{\underline{H}}_{1}, \underline{\underline{Q}}_{1} \stackrel{\text { def }}{=} \underline{\underline{H}}_{1} \underline{\underline{\hat{1}}} \underline{\underline{H}}_{0}+\left(\underline{\underline{H}}_{0}-A_{J: 1} \underline{\underline{H}}_{1}\right) \underline{\underline{\hat{1}}}_{\underline{\underline{H}}} \\
& \underline{\underline{K}}_{3} \stackrel{\text { def }}{=} 2 \underline{\underline{Q}}{ }_{2} \underline{\underline{\underline{1}}} \underline{\underline{H}}_{1}, \underline{\underline{K}}_{2} \stackrel{\text { def }}{=} 2 \underline{\underline{Q}} \underline{\underline{\hat{1}}}_{2} \underline{\underline{H}}_{0}+\left(\underline{\underline{Q}}_{1}-A_{J: 1} \underline{\underline{Q}}_{2}\right) \underline{\underline{\hat{1}}}_{\underline{\underline{H}}} \underline{\underline{H}}_{1} \\
& \underline{\underline{V}}_{2} \stackrel{\text { def }}{=}-\underline{\underline{H}}_{1}\left(\underline{\underline{A}}_{D: 1} \underline{\underline{\rho}}^{\#}+\underline{\underline{D}}_{1}^{\#}\right) \underline{\underline{H}}_{1} \text {, } \\
& \underline{\underline{V}}_{1} \stackrel{\text { def }}{=}\left\{\underline{\underline{H}}_{1}\left(\underline{\underline{A}}_{D: 2} \underline{\underline{\rho}}^{\#}+\underline{\underline{A}}_{D: 1} \underline{\underline{D}}_{1}^{\#}\right)-\underline{\underline{H}}_{0}\left(\underline{\underline{A}}_{D: 1} \underline{\underline{\rho}}^{\#}+\underline{\underline{D}}_{1}^{\#}\right)\right\} \underline{\underline{H}}_{1}-\underline{\underline{H}}_{1}\left(\underline{\underline{A}}_{D: 1} \underline{\underline{\rho}}^{\#}+\underline{\underline{D}}_{1}^{\#}\right) \underline{\underline{H}}_{0} \text {, } \\
& \underline{\underline{W}}_{2} \stackrel{\text { def }}{=}-2 \underline{\underline{H}}_{0}\left(\underline{\underline{A}}_{D: 1} \underline{\underline{\rho}}^{\#}+\underline{\underline{D}}_{1}^{\#}\right) \underline{\underline{V}}_{2}+\underline{\underline{H}}_{1}\left\{-2\left(\underline{\underline{A}}_{D: 1} \underline{\underline{\rho}}^{\#}+\underline{\underline{D}}_{1}^{\#}\right) \underline{\underline{V}}_{1}+2\left(\underline{\underline{A}}_{D: 2} \underline{\underline{\rho}}^{\#}+\underline{\underline{A}}_{D: 1} \underline{\underline{D}}_{1}^{\#}\right) \underline{\underline{V}}_{2}\right. \\
& \left.+\left(\underline{\underline{A}}_{D: 2} \underline{\underline{\rho}}_{D} \underline{\underline{\rho}}^{\#}+2 \underline{\underline{A}}_{D: 1} \underline{\underline{\rho}}_{D} \underline{\underline{D}}_{1}^{\#}+\underline{\underline{D}}_{2}^{\#}\right) \underline{\underline{H}}_{1}\right\} \\
& \underline{\underline{R}}_{3} \stackrel{\text { def }}{=} \underline{\underline{V}}_{2} \hat{\underline{1}} \underline{\underline{H}}_{1}+\underline{\underline{H}}_{1} \hat{\underline{1}} \underline{\underline{V}}_{2} \text { ， } \\
& \underline{\underline{R}}_{2} \stackrel{\text { def }}{=}\left(\underline{\underline{V}}_{2} \underline{\underline{1}} \underline{\underline{H}}_{0}+\underline{\underline{H}}_{0} \hat{\underline{1}} \underline{\underline{V}}_{2}\right)+\left(\underline{\underline{V}}_{1} \underline{\underline{\hat{1}}} \underline{\underline{H}}_{1}+\underline{\underline{H}}_{1} \underline{\underline{\hat{1}}} \underline{\underline{V}}_{1}\right)-A_{J: 1}\left(\underline{\underline{V}}_{2} \underline{\underline{1}} \underline{\underline{H}}_{1}+\underline{\underline{H}}_{1} \hat{\underline{1}} \underline{\underline{V}}_{2}\right)
\end{aligned}
$$$$
\underline{\underline{W}}_{3} \stackrel{\text { def }}{=}-2 \underline{\underline{H}}_{1}\left(\underline{\underline{A}}_{D: 1} \underline{\underline{\rho}}^{\#}+\underline{\underline{D}}_{1}^{\#}\right) \underline{\underline{V}}_{2} \text {, }
$$$$
-\left\{\rho(J) A_{J: 1}+E\left[D_{J}\right]\right\} \underline{\underline{H}}_{1} \underline{\underline{\hat{1}}}_{\underline{\underline{H}}} \text {. }
$$

Lemma A.3 As $t \rightarrow \infty$,
a) $E[N(t)]=\underline{p}^{\top}(0)\left(\underline{\underline{L}}_{1} t+\underline{\underline{L}}_{0}\right) \underline{1}+o(1)$
b) $E\left[N^{2}(t)\right]=\underline{p}^{\top}(0)\left(\underline{\underline{S}}_{2} t^{2}+\underline{\underline{S}}_{1} t\right) \underline{1}+o(t)$
c) $E\left[Z^{2}(t)\right]=\underline{p}^{\top}(0)\left(\underline{\underline{T}}_{2} t^{2}+\underline{\underline{T}}_{1} t\right) \underline{1}+o(t)$
d) $E[N(t) Z(t)]=\underline{p}^{\top}(0)\left(\underline{\underline{U}}_{2} t^{2}+\underline{\underline{U}}_{1} t\right) \underline{1}+o(t)$

where

$$
\begin{aligned}
& \underline{\underline{L}}_{1} \stackrel{\text { def }}{=} \underline{\underline{Q}}_{2} \underline{\underline{A}}_{D: 1}, \underline{\underline{L}}_{0} \stackrel{\text { def }}{=} \underline{\underline{Q}}_{1} \underline{\underline{A}}_{D: 1}-\frac{1}{2} \underline{\underline{Q}}_{2} \underline{\underline{A}}_{D: 2}, \\
& \underline{\underline{S}}_{2} \stackrel{\text { def }}{=} \underline{\underline{K}}_{3} \underline{\underline{A}}_{D: 1}, \underline{\underline{S}}_{1} \stackrel{\text { def }}{=}\left(\underline{\underline{K}}_{2}+\underline{\underline{Q}}_{2}\right) \underline{\underline{A}}_{D: 1}-\frac{1}{2} \underline{\underline{K}}_{3} \underline{\underline{A}}_{D: 2},
\end{aligned}
$$




$$
\begin{aligned}
& \underline{\underline{T}}_{2} \stackrel{\text { def }}{=} \underline{\underline{W}}_{3} \underline{\underline{A}}_{D: 1}, \underline{\underline{T}}_{1} \stackrel{\text { def }}{=} \underline{\underline{W}}_{2} \underline{A}_{D: 1}-\frac{1}{2} \underline{\underline{W}}_{3} \underline{\underline{A}}_{D: 2}-\underline{\underline{V}}_{2} \underline{\rho}_{D} \underline{\underline{A}}_{D: 2} \\
& \underline{\underline{U}}_{2} \stackrel{\text { def }}{=}-\underline{\underline{R}}_{3} \underline{\underline{A}}_{D: 1}, \underline{\underline{U}}_{1} \stackrel{\text { def }}{=}-\underline{\underline{R}}_{2} \underline{\underline{A}}_{D: 1}+\frac{1}{2} \underline{\underline{R}}_{3} \underline{\underline{A}}_{D: 2}+\frac{1}{2} \underline{\underline{Q}}_{2} \underline{\rho}_{D} \underline{\underline{A}}_{D: 2}
\end{aligned}
$$

\section{References}

[1] E. Chinlar: Markov renewal theory. Advances in Applied Probability, 1 (1969), 123-187.

[2] E. Çinlar: Markov renewal theory: A survey. Management Science, 21-7 (1975), 727752.

[3] E. Çinlar: Introduction to Stochastic Processes (Prentice-Hall, 1975).

[4] D.R. Cox: Renewal Theory (Methuen, 1962).

[5] R. Howard: Dynamic Probabilistic Systems Vol I, II (Wiley, 1971).

[6] J.J. Hunter: The moments of Markov renewal processes. Advances in Applied Probability, 1 (1969), 188-210.

[7] N. Igaki, U. Sumita and M. Kowada: Analysis of Markov renewal shock models. Journal of Applied Probability, 32 (1995), 821-831.

[8] W.S. Jewell: Markov renewal programming, I, formulation, finite return model. Operations Research, 11 (1963), 938-948.

[9] W.S. Jewell: Limiting Covariance in Markov Renewal Processes (ORC 64-16, Univ. California, Berkeley, 1964).

[10] W.S. Jewell: Fluctuations of a renewal reward process. SIAM Journal on Mathematical Analysis, 19 (1967), 309-329.

[11] R.W. Keener: Renewal theory for Markov chains on the real line. The Annals of Probability, 10-4 (1982), 942-954.

[12] J. Keilson: On the matrix renewal function for Markov renewal processes. The Annals of Mathematical Statistics, 40 (1969), 1901-1907.

[13] J. Keilson and S.S. Rao: A process with chain dependent growth rate. Journal of Applied Probability, 3 (1970), 699-711.

[14] J. Keilson and S.S. Rao: A process with chain dependent growth rate. part II : the ruin and ergodic problems. Advances in Applied Probability, 3 (1971), 315-338.

[15] M. Kijima and U. Sumita: A useful generalization of renewal theory : counting processes governed by non-negative Markovian increments. Journal of Applied Probability, 23 (1986), 71-88.

[16] P. Lévy: Processus Semi-Markoviens (Proc. Int. Congress. Math. III, Amsterdam, 1954).

[17] Y. Masuda: Dynamic analysis of computer and communication systems: semi-Markov approach (Ph.D. thesis, William E. Simon Graduate School of Business Administration, University of Rochester, New York, 1987).

[18] Y. Masuda: Partially Observable semi-Markov reward processes. Journal of Applied Probability, 30 (1993), 548-560.

[19] Y. Masuda and U. Sumita: A multivariate reward process defined on a semi-Markov process and its first passage time distributions. Journal of Applied Probability, 28 (1991), 287-302.

[20] R.A. McLean and M.F. Neuts: The integral of a step function defined on a semi-Markov process. SIAM Journal on Applied Mathematics, 15-3 (1967), 726-737. 
[21] R.A. Moore and R. Pyke: Estimation of transition distributions of a Markov renewal processes. The Annals of Mathematical Statistics, 20 (1968), 411-424.

[22] R. Pyke: Markov renewal processes : definitions and preliminary properties. The Annals of Mathematical Statistics, 32 (1961), 1231-1242.

[23] R. Pyke: Markov renewal processes with finitely many states. The Annals of Mathematical Statistics, 32 (1961), 1243-1259.

[24] R. Pyke and R. Schaufele: Limit theorems for Markov renewal processes. The Annals of Mathematical Statistics, 35 (1964), 1746-1764.

[25] W.L. Smith: Regenerative stochastic processes. Proceedings of the Royal Society of London Series A, 232 (1955), 6-31.

[26] U. Sumita, P. Kubat and Y. Masuda: Dynamic performance analysis of communication/computer systems with highly reliable components. Probability in the Engineering and Information Sciences, 2 (1988), 185-213.

[27] U. Sumita and Y. Masuda: An alternative approach to the analysis of finite semiMarkov and related processes. Stochastic Models, 3-1 (1987), 67-87.

[28] U. Sumita, Y. Masuda and P. Kubat: Development of performance functions for communication/computer systems and its application to dynamic performance analysis. Microelectronics and Reliability, 29-6 (1989), 973-991.

[29] L. Takács: Some investigations concerning recurrent stochastic processes of a certain type. Maygyar Tud. Akad. Mad. Kutató int. Kozl, 3 (1954), 115-128.

Ushio Sumita

Graduate School of Systems and Information Engineering

University of Tsukuba

1-1-1 Tennoudai Tsukuba

Ibaraki 305-8573, Japan

E-mail: sumita@sk.tsukuba.ac.jp 